\title{
Effect of Green Tea Supplementation on Antioxidant Status in Adults: A Systematic Review and Meta-Analysis of Randomized Clinical Trials
}

\author{
Niloufar Rasaei ${ }^{1}$, Omid Asbaghi ${ }^{2}$, Mahsa Samadi ${ }^{1}$, Leila Setayesh ${ }^{1} \mathbb{D}$, Reza Bagheri ${ }^{3}$, Fatemeh Gholami ${ }^{1}$, \\ Neda Soveid ${ }^{1}$, Krista Casazza ${ }^{4}$, Alexei Wong ${ }^{5}$, Katsuhiko Suzuki ${ }^{6, * \mathbb{D}}$ and Khadijeh Mirzaei ${ }^{1, *}$
}

1 Department of Community Nutrition, School of Nutritional Sciences and Dietetics, Tehran University of Medical Sciences, Tehran 14176-13151, Iran; n.rasaei71@gmail.com (N.R.); mahsa_samadi@ymail.com (M.S.); setayesh.leila@yahoo.com (L.S.); gholami_fghlm67@yahoo.com (F.G.); neda.soveid@gmail.com (N.S.)

2 Cancer Research Center, Shahid Beheshti University of Medical Sciences, Tehran 14167-53955, Iran; omid.asbaghi@gmail.com

3 Department of Exercise Physiology, University of Isfahan, Isfahan 81746-73441, Iran; will.fivb@yahoo.com

4 Marieb College of Health and Human Services, Florida Gulf Coast University, Fort Myers, FL 33965, USA; krista.casazza@gmail.com

5 Department of Health and Human Performance, Marymount University, Arlington, VA 22207, USA; awong@marymount.edu

check for

updates

Citation: Rasaei, N.; Asbaghi, O.; Samadi, M.; Setayesh, L.; Bagheri, R.; Gholami, F.; Soveid, N.; Casazza, K.; Wong, A.; Suzuki, K.; et al. Effect of Green Tea Supplementation on Antioxidant Status in Adults: A Systematic Review and MetaAnalysis of Randomized Clinical Trials. Antioxidants 2021, 10, 1731. https://doi.org/10.3390/antiox 10111731

\section{Academic Editors: Eduardo}

Guerra-Hernández and Stanley Omaye

Received: 27 September 2021

Accepted: 27 October 2021

Published: 29 October 2021

Publisher's Note: MDPI stays neutral with regard to jurisdictional claims in published maps and institutional affiliations.

Copyright: (C) 2021 by the authors Licensee MDPI, Basel, Switzerland. This article is an open access article distributed under the terms and conditions of the Creative Commons Attribution (CC BY) license (https:/ / creativecommons.org/licenses/by/ $4.0 /)$.
6 Faculty of Sport Sciences, Waseda University, 2-579-15 Mikajima, Tokorozawa 359-1192, Japan

* Correspondence: katsu.suzu@waseda.jp (K.S.); mirzaei_kh@sina.tums.ac.ir (K.M.)

Abstract: It is well-established that green tea supplementation has antioxidant properties. However, whether green tea supplementation leads to oxidative stress reduction remains unclear, as clinical investigations on this subject have yielded inconsistent outcomes. Consequently, we aimed to determine the effects of green tea supplementation on oxidative stress in adults. A systematic search of English language publications up to 21 August 2021 was carried out in PubMed, Scopus, Embase, and ISI Web of Science, utilizing pertinent keywords. These searches included randomized controlled trials (RCTs) evaluating the relationship between green tea supplementation, malondialdehyde (MDA), and total antioxidant capacity (TAC) in adults. A random-effects model was used to estimate the weighted mean difference (WMD) and 95\% confidence intervals (95\% CI). Meta-regression and non-linear dose-response analyses were performed to investigate the association between the dosage of green tea (mg/day) and the duration of the intervention (weeks) with pooled effect size. Sixteen RCTs with seventeen arms including 760 participants met the inclusion criteria. Our results indicated that green tea supplementation had significant effects on TAC (weighted mean difference [WMD]: $0.20 \mathrm{mmol} / \mathrm{L} ; 95 \%$ CI: $0.09,0.30, p<0.001)$ and significant heterogeneity between studies $\left(\mathrm{I}^{2}=98.6 \%\right.$, $p<0.001$ ), which was largely related to gender and body mass index (BMI). Subgroup analysis in TAC identified a significant relationship except with low dose supplementation and obese individuals. No relationship between MDA and green tea supplementation was observed in any subgroups; however, meta-regression analysis revealed a linear inverse association between the dosage and significant change in MDA ( $r=-2117.18, p=0.017)$. Our outcomes suggest that green tea supplementation improves TAC and affects MDA based on the dose of the intervention in adults. Future RCTs with longer durations are needed to expand our findings.

Keywords: green tea; oxidative stress; total antioxidant capacity; malondialdehyde

\section{Introduction}

All metabolic processes generate reactive oxygen species (ROS), which can induce oxidative damage to tissue and cell constituents such as lipids, proteins, and nucleic acids [1,2] when left unbalanced. Indeed, the overproduction and accumulation of large amounts of ROS lead to oxidative stress [3-5], which represents a major contributor to 
the risk, onset, and progression of chronic diseases [6-12]. A key component of oxidative stress implicated in adverse health effects is lipid peroxidation of polyunsaturated fatty acids and the subsequent production of malondialdehyde (MDA) (a major indicator of lipid oxidation) [9]. Therefore, interventions to intensify the body's antioxidant response to counteract oxidative stress and MDA production are desperately needed.

Natural dietary polyphenolic compounds are potent antioxidants that decrease oxidative stress and protect the body against several oxidative stress-associated diseases [13,14]. Among the common sources of natural dietary polyphenol in foods and beverages is green tea (originate from the leaves of Camellia sinensis), which has high antioxidant properties related to various compounds such as phenolics and flavonoids, and flavonols [15-17]. In fact, the major polyphenols with antioxidant properties in green tea are epicatechin (EC), epigallocatechin-3-gallate (EGCG), epicatechin-3-gallate (ECG), epigallocatechin (EGC), and gallocatechin gallate (GCG), which are also called catechins [18]. While some studies have reported that EGCG and other catechins in green tea increase the activity of antioxidant enzymes, decrease indices of oxidative damage and prevent and treat some diseases [19-21], there are equivocal effects on markers of oxidative stress in diabetes, metabolic syndrome, $\beta$-thalassemia major, and obesity $[9,13,22-25]$. Methodological issues such as non-randomization, different outcome measures, short duration of treatment, low study quality, and population heterogeneity have precluded a comprehensive understanding of the extent to which green tea supplementation may exert a beneficial effect on oxidative stress. Further, the potential effectiveness of green tea supplementation in mitigating oxidative stress among adults with pre-existing inflammatory conditions has not been clarified. Thus, we performed a systematic review and meta-analysis of randomized controlled trials (RCTs) to evaluate the relationship between green tea supplementation and oxidative stress markers, as assessed by the MDA and total antioxidant capacity (TAC), in adults with and without inflammatory health conditions.

\section{Materials and Methods}

Findings from this systematic review and meta-analysis were reported based on the Preferred Reporting Items of Systematic Reviews and Meta-Analysis (PRISMA) guideline [26]. The PICOS model was used to prepare the question: population (all individuals), intervention (green tea), comparison (studies which had control group), outcome (studies that reported two markers of oxidative stress including MDA and TAC), and study design (RCT, Registration code: CRD42021240026) [27].

\subsection{Search Strategy}

We conducted database searches of PubMed, Scopus, Embase, and ISI web of science from inception to 21 August 2021. All relevant studies written in the English language were included using. Medical subject headings (MeSH) and text words. Search words included:

"green tea" OR "green tea extract" OR "green tea extract AR25" OR "catechin" OR "catechins" OR "EGCG" OR "Camellia sinensis" OR "tea polyphenols" OR "catechinic acid" OR "acid catechinic" OR "sinensis Camellia" OR "Thea sinensis" OR "sinensis Thea" OR "tea polyphenols" AND "oxidative stress" OR malondialdehyde OR MDA OR glutathione OR GSH OR "total antioxidant capacity" OR TAC OR "total antioxidant status" OR TAS AND.

intervention OR "intervention study" OR "intervention studies" OR "controlled trial" OR randomized OR randomized OR random OR randomly OR placebo OR assignment OR "clinical trial" OR trial OR assignment OR "randomized controlled trial" OR "randomized clinical trial" OR RCT OR blinded OR "double blind" OR "double blinded" OR trial OR "clinical trial" OR trials OR "pragmatic clinical trial" OR "cross-over studies" OR "cross-over" OR "cross-over study" OR parallel OR "parallel study" OR "parallel trial". Reference lists of the included studies were manually checked to identify additional articles. Unpublished documents and grey literature such as thesis, patents, and conference papers were not included. 


\subsection{Eligibility Criteria}

Studies were included if they met the following criteria: RCT design, population aged $>18$ years, and reported MDA and/or TAC in both the intervention and placebo groups. Studies with duplicated data, non-RCT design, animal studies, studies that assess the effects of green tea supplementation and other interventions, and those with insufficient data for the outcomes of interest or did not meet inclusion criteria of our meta-analysis were excluded.

\subsection{Data Extraction}

Two independent researchers extracted data and evaluated any disagreement was resolved through open discussion with a third independent investigator (KhM). First author's name, year of publication, study location, duration and design, gender, mean age and mean body mass index (BMI) of population, the health status of participants, number of participants in each group, type and dose of green tea, and results (means and standard deviations for MDA and TAC before and after intervention) were extracted from the full text of included studies.

\subsection{Quality Assessment}

Assessment of study quality each included study was precisely evaluated for quality by the Cochrane scoring system [28] of 7 points based on the following criteria: (1) random sequence generation, (2) allocation concealment, (3) blinding of participants and personnel, (4) blinding of outcome assessment, (5) incomplete outcome data, (6) selective reporting, (7) other biases. Three scores of $\mathrm{L}, \mathrm{H}$, and $\mathrm{U}$ could be given to each aforementioned item, which is interpreted as low risk, high risk, and unknown risk, respectively. Two studies had low random sequence generation [29,30]. Allocation concealment reported in 15 studies $[4,5,10,13,23,29-37]$. Moreover, 3 trials had a high risk of bias regarding blinding of participants, personnel $[9,24,31]$, and 3 trials had low-risk outcome assessors [22,29,34]. Selective reporting was considered as low risk in one trial [10]. All of the studies showed a low risk of bias based on incomplete outcome data. Other sources of bias were low in 10 trials $[5,10,13,22,29,30,32,34,35,37]$.

\subsection{Statistical Analysis}

This meta-analysis was performed using STATA software, version 14.0 (Stata Corp. LP, College Station, TX, USA). The effect of green tea supplementation on oxidative stress (MDA and TAC) was estimated by pooling mean and standard deviation (SD) values of the baseline and final of the studies in both intervention and placebo groups. Missing SDs for changes were calculated utilizing the following formula: SD change $=$ square root [(SD baseline ${ }^{2}+\mathrm{SD}$ final $\left.^{2}\right)-(2 \times \mathrm{R} \times \mathrm{SD}$ baseline $\times \mathrm{SD}$ final $\left.)\right]$, if the studies did not report mean and SD. We considered a correlation coefficient of 0.9 as the R-value of the mentioned formula [38]. We used the following formula to calculate standard errors (SE) from $\mathrm{SD}: \mathrm{SE} \sqrt{ } \mathrm{n}$. If a study provided medians (interquartile ranges), we converted them to means (SD) as described by Hozo and colleagues [39]. If the study results were reported graphically, we used GetData Graph Digitizer software to estimate mean (SD). At first, a fixed-effect model was performed to pool the data. A random-effects model was used to estimate the weighted mean difference (WMD) and $95 \%$ confidence $(95 \% \mathrm{CI})$. Heterogeneity among studies was assessed with the $\mathrm{Q}$ test and the $\mathrm{I}^{2}$ index statistic. If $p<0.1$ and $\mathrm{I}^{2}>50 \%$, it was considered that heterogeneity existed among studies. If $p>0.1$ and $\mathrm{I}^{2}<50 \%$, fixed-effect models would be applied. Sensitive analysis and subgroup analysis were performed to evaluate the source of heterogeneity and verified the stability of results. In the sensitivity analysis, one study was omitted at each turn to evaluate the influence of each study on the results. Subgroup analysis was performed by stratifying study type, region, study quality score, and maximum category. Contour-enhanced funnel plots with an Egger linear regression test and a Begg rank correlation test were used to evaluate the potential publication bias. Meta-regression and non-linear dose-response 
analyses were performed to investigate the association between the dosage of green tea (mg/day) and the duration of the intervention (weeks) with pooled effect size. Because all the data used for analyses were extracted from the published studies, ethical approval and informed consent were not necessary.

\section{Results}

\subsection{Study Selection}

We found 3204 publications in Scopus, PubMed, Embase, and ISI web of science in the initial search. Of these, eight hundred seventy-four articles were duplicated. Thus, a total of 2330 articles were assessed for the title and abstract screening. After screening of title and abstract, 2288 unrelated studies were discarded due to primary evaluation of inclusion criteria: unrelated title $(n=1729)$, review $(n=123)$, and animal study (436). Consequently, 42 studies were retrieved for full-text review, 26 of which were excluded due to: lack of sufficient data and the use of two or more interventions at the same time $[2,21,22,24,30,34,40-61]$. Finally, sixteen RCTs with one double-arm study $[4,5,9,10,13,22,24,29,37]$ were eligible for this systematic review and meta-analysis. The flow chart of the literature search is shown in Figure 1.

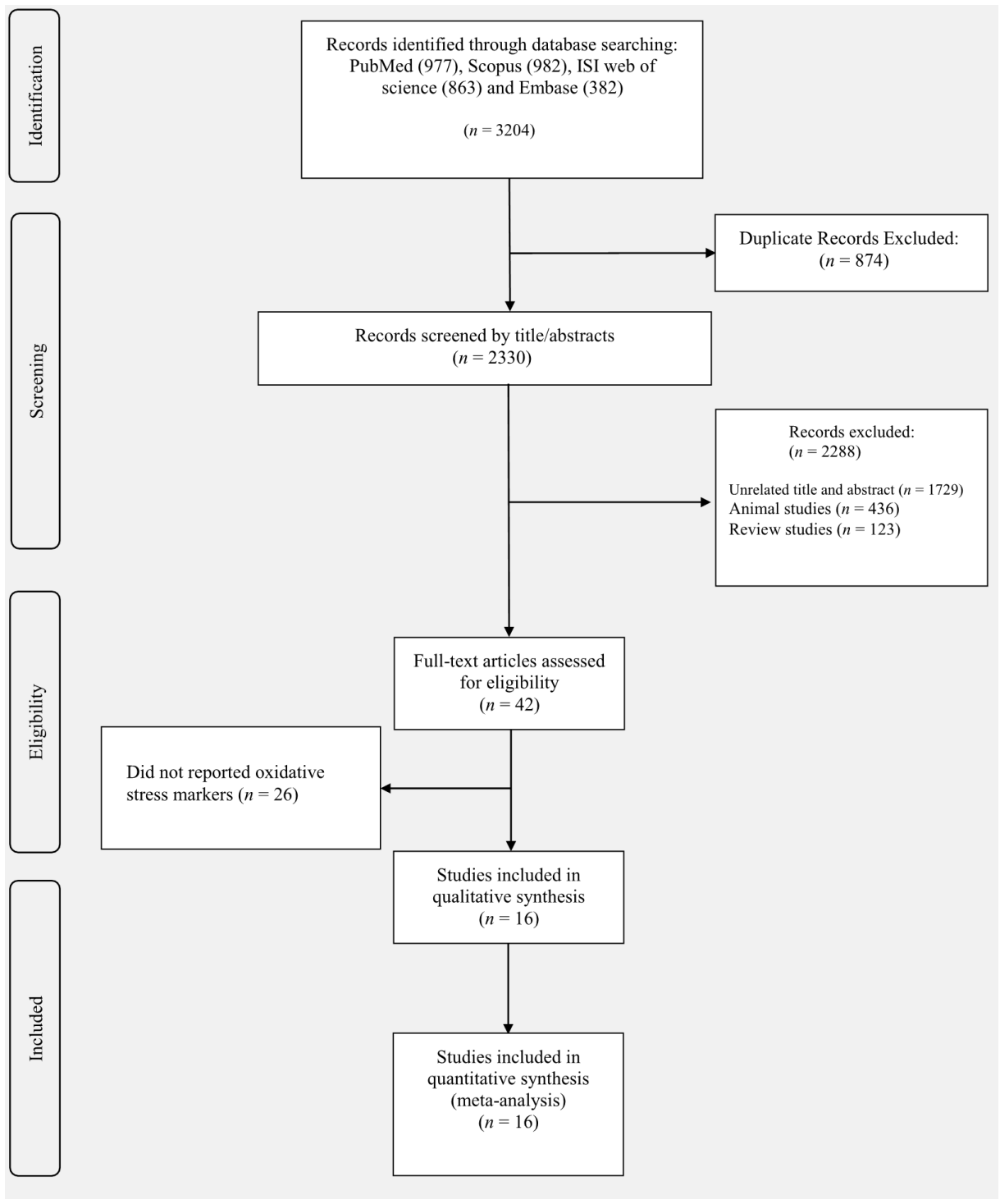

Figure 1. The flow chart of the literature search. 


\subsection{Study Characteristics}

Sixteen RCTs with one double-arm study assessing the effects of green tea supplementation on oxidative stress were identified. Included studies carried out in various countries such as Iran [5,9,22,24,33,36,37], Poland [4,29,31,32], Lithuania [13,31], Taiwan [34,35] and Brazil [10]. Publication dates ranged from 2009 and 2020. The follow-up period ranged from 2 weeks to 36 weeks. The sample size of the included studies ranged from eight to forty-eight participants. Some studies enrolled only females [35] or males [4,5,9,31,34,36], and the rest of included studies involved both genders [10,13,22,24,29,32,33,37], and one of them was unclear [31]. Type of intervention administration were green tea $[9,22,24,34,35]$ and green tea extract $[4,5,9,10,13,29,32,34,36,37]$. Three studies were conducted on obese individuals $[9,29,32]$, four studies on healthy individuals $[4,5,31,34,36]$, five studies were conducted on diabetics [10,13,24,30,33,37], one study on $\beta$-thalassemia major [29] and one study on hypercholesterolemic participants [35]. The studies were performed in participants with different baseline BMI; five studies carried out in participants under $25 \mathrm{~kg} / \mathrm{m}^{2}[4,5,22,31,34,36]$, eight studies over than $25 \mathrm{~kg} / \mathrm{m}^{2}[9,10,24,29,30,32,33,35,37]$ and one study did not report BMI [13]. All studies were parallel RTCs [4,5,9,10,13,22,24,29,37]. The summary of the characteristics of the included studies is indicated in Table 1. Results from the quality assessment are shown in Table 2.

\subsection{Meta-Analysis}

\subsubsection{Effect of Green Tea Supplementation on TAC}

Overall, 16 studies with seventeen arms evaluated the effect of green tea supplementation on TAC. Pooled effect size from random effect model showed a significant increasing effect of green tea supplementation on TAC (WMD: $0.20 \mathrm{mmol} / \mathrm{L} ; 95 \% \mathrm{CI}$ : $0.09,0.30, p<0.001)$. There was significant heterogeneity between studies $\left(\mathrm{I}^{2}=98.6 \%\right.$, $p<0.001$ ) (Figure 2A). However, after subgroup analysis we observed significant effect of green tea supplementation on TAC in studies of less (WMD: $0.43 \mathrm{mmol} / \mathrm{L} ; 95 \% \mathrm{CI}: 0.10$, $0.75, p=0.010$ ) or more than 8 weeks (WMD: $0.08 \mathrm{mmol} / \mathrm{L} ; 95 \%$ CI: $0.02,0.13, p=0.004$ ). Similar significant effects were also detected in green tea extract (WMD: $0.23 \mathrm{mmol} / \mathrm{L} ; 95 \%$ CI: $0.03,0.42, p=0.019)$ and brewed green tea (WMD: $0.07 \mathrm{mmol} / \mathrm{L} ; 95 \% \mathrm{CI}: 0.03,0.10$, $p<0.001$ ) and male (WMD: $0.38 \mathrm{mmol} / \mathrm{L} ; 95 \%$ CI: 0.07, 0.69, $p=0.003$ ), female (WMD: $0.05 \mathrm{mmol} / \mathrm{L} ; 95 \%$ CI: 0.04, 0.05, $p=0.015$ ) and both sexes (WMD: $0.12 \mathrm{mmol} / \mathrm{L} ; 95 \%$ CI: $0.08,0.17, p<0.001)$. In addition, sub-group analysis based on dose showed that green tea supplementation for $>400 \mathrm{mg} /$ day had significant effect on TAC (WMD: $0.29 \mathrm{mmol} / \mathrm{L}$; 95\% CI: $0.09,0.49, p=0.004)$. In addition, subgroup analysis demonstrated that both healthy (WMD: $0.08 \mathrm{mmol} / \mathrm{L} ; 95 \%$ CI: $0.02,0.13, p=0.004$ ) and unhealthy status (WMD: $0.43 \mathrm{mmol} / \mathrm{L} ; 95 \%$ CI: $0.10,0.75, p=0.010$ ) significantly affect TAC. Sub-group analysis based on BMI showed that those with BMI $=18.5-24.9$ and 25-29.9 had significant effect on TAC respectively (WMD: $0.38 \mathrm{mmol} / \mathrm{L} ; 95 \%$ CI: 0.08, 0.68, $p=0.011$ ) (WMD: $0.11 \mathrm{mmol} / \mathrm{L}$; 95\% CI: 0.07, 0.15, $p<0.001$ ) (Table 3). 
Table 1. Characteristic of included studies in meta-analysis.

\begin{tabular}{|c|c|c|c|c|c|c|c|c|c|c|c|c|c|c|c|}
\hline \multirow[b]{2}{*}{ Author } & \multirow[b]{2}{*}{$\begin{array}{l}\text { Publication } \\
\text { Year }\end{array}$} & \multirow[b]{2}{*}{ Country } & \multirow{2}{*}{$\begin{array}{l}\text { Study } \\
\text { Design }\end{array}$} & \multirow[b]{2}{*}{ Participant } & \multirow[b]{2}{*}{ Sex } & \multirow{2}{*}{$\begin{array}{c}\text { Trial } \\
\text { Duration } \\
\text { (Week) }\end{array}$} & \multicolumn{2}{|c|}{ Means Age } & \multicolumn{2}{|c|}{ Means BMI } & \multicolumn{3}{|c|}{ Intervention } & \multicolumn{2}{|c|}{ Sample Size } \\
\hline & & & & & & & IG & CG & IG & CG & $\begin{array}{l}\text { Treatment } \\
\text { Group }\end{array}$ & $\begin{array}{c}\text { Green Tea Dose } \\
(\mathrm{mg})\end{array}$ & $\begin{array}{l}\text { Control } \\
\text { Group }\end{array}$ & IG & CG \\
\hline $\begin{array}{c}\text { Azizbeigi et al. } \\
2009\end{array}$ & 2009 & Iran & $\mathrm{RCT}$ & Obese men & $\mathrm{M}$ & 8 & 23.9 & 22.8 & 31.8 & 30.8 & $\begin{array}{c}\text { Green tea } \\
\text { extract }\end{array}$ & $500 \mathrm{mg}$ & $\begin{array}{l}500 \mathrm{mg} \\
\text { sucrose }\end{array}$ & 10 & 10 \\
\hline $\begin{array}{c}\text { Jowko et al. } \\
2011\end{array}$ & 2011 & Poland & $\mathrm{DB} / \mathrm{R} / \mathrm{PC}$ & $\begin{array}{c}\text { Healthy } \\
\text { individuals }\end{array}$ & $\mathrm{M}$ & 4 & 21.5 & 21.2 & 23.85 & 23.31 & $\begin{array}{c}\text { Green tea } \\
\text { extract }\end{array}$ & $\begin{array}{c}640 \mathrm{mg} \text { of } \\
\text { polyphenols }\end{array}$ & Maltodextrin & 17 & 18 \\
\hline $\begin{array}{l}\text { Bogdanski } \\
\text { et al. } 2012\end{array}$ & 2012 & Poland & $\mathrm{DB} / \mathrm{R} / \mathrm{PC}$ & $\begin{array}{c}\text { Obese, } \\
\text { hypertensive } \\
\text { patients }\end{array}$ & $\mathrm{F} / \mathrm{M}$ & 12 & 49.2 & 51.5 & 32.5 & 33.9 & $\begin{array}{c}\text { Green tea } \\
\text { extract }\end{array}$ & $379 \mathrm{mg}$ & $\begin{array}{l}\text { Pure micro- } \\
\text { crystalline } \\
\text { cellulose }\end{array}$ & 28 & 28 \\
\hline $\begin{array}{l}\text { Suliburska } \\
\text { et al. } 2012\end{array}$ & 2012 & Poland & $\mathrm{DB} / \mathrm{R} / \mathrm{PC}$ & Obese patients & $\mathrm{F} / \mathrm{M}$ & 12 & 48.56 & 52.26 & 32.07 & 33.45 & $\begin{array}{c}\text { Green tea } \\
\text { extract }\end{array}$ & $379 \mathrm{mg}$ & $\begin{array}{l}\text { Pure micro- } \\
\text { crystalline } \\
\text { cellulose }\end{array}$ & 23 & 23 \\
\hline $\begin{array}{c}\text { Mousavi et al. } \\
2013 \text { (A) }\end{array}$ & 2013 & Iran & $\mathrm{RCT}$ & T2DM patients & $\mathrm{F} / \mathrm{M}$ & 8 & 54.6 & 52 & 27.4 & 28.1 & Green tea & 4 cup $=10,000 \mathrm{mg}$ & - & 26 & 14 \\
\hline $\begin{array}{l}\text { Mousavi et al. } \\
2013 \text { (B) }\end{array}$ & 2013 & Iran & $\mathrm{RCT}$ & T2DM patients & $\mathrm{F} / \mathrm{M}$ & 8 & 56.2 & 52 & 28.1 & 28.1 & Green tea & $2 \mathrm{cup}=5000 \mathrm{mg}$ & - & 25 & 14 \\
\hline $\begin{array}{l}\text { Lasaite et al. } \\
2014 \text { (A) }\end{array}$ & 2014 & Lithuania & $\mathrm{DB} / \mathrm{R} / \mathrm{PC}$ & T2DM patients & $\mathrm{F} / \mathrm{M}$ & 36 & 57.2 & 56.8 & NR & NR & $\begin{array}{c}\text { Green tea } \\
\text { extract }\end{array}$ & 400 & $\begin{array}{l}\text { Microcrystalline } \\
\text { cellulose }\end{array}$ & 17 & 14 \\
\hline $\begin{array}{l}\text { Lasaite et al. } \\
2014 \text { (B) }\end{array}$ & 2014 & Lithuania & $\mathrm{DB} / \mathrm{R} / \mathrm{PC}$ & T2DM patients & $\mathrm{F} / \mathrm{M}$ & 36 & 57.2 & 56.8 & NR & NR & $\begin{array}{c}\text { Green tea } \\
\text { extract }\end{array}$ & 600 & $\begin{array}{l}\text { Microcrystalline } \\
\text { cellulose }\end{array}$ & 17 & 14 \\
\hline $\begin{array}{c}\text { Spadiene et al. } \\
2014 \text { (A) }\end{array}$ & 2014 & Lithuania & $\mathrm{DB} / \mathrm{R} / \mathrm{PC}$ & T2DM patients & $\mathrm{nr}$ & 36 & 62.18 & 62.18 & 35.23 & 34.98 & $\begin{array}{c}\text { Green tea } \\
\text { extract }\end{array}$ & 400 & $\begin{array}{l}\text { Microcrystalline } \\
\text { cellulose }\end{array}$ & 20 & 25 \\
\hline $\begin{array}{l}\text { Spadiene et al. } \\
2014 \text { (B) }\end{array}$ & 2014 & Lithuania & $\mathrm{DB} / \mathrm{R} / \mathrm{PC}$ & T2DM patients & $\mathrm{nr}$ & 36 & 62.18 & 62.18 & 35.23 & 34.98 & $\begin{array}{c}\text { Green tea } \\
\text { extract }\end{array}$ & 600 & $\begin{array}{l}\text { Microcrystalline } \\
\text { cellulose }\end{array}$ & 20 & 25 \\
\hline $\begin{array}{c}\text { Mozaffari- } \\
\text { Khosravi et al. } \\
2014\end{array}$ & 2014 & Iran & $\mathrm{RCT}$ & $\begin{array}{c}\text { Type } 2 \text { Diabetes } \\
\text { Mellitus }\end{array}$ & $\mathrm{F} / \mathrm{M}$ & 4 & 52.2 & 52.1 & 28 & 28.3 & Green tea & $450 \mathrm{~mL}$ & Sour tea & 48 & 46 \\
\hline $\begin{array}{l}\text { Kuo et al. } \\
2015\end{array}$ & 2015 & Taiwan & $\mathrm{DB} / \mathrm{PC}$ & $\begin{array}{c}\text { Healthy } \\
\text { individuals }\end{array}$ & $\mathrm{M}$ & 4 & 20 & 20 & 21.95 & 23.55 & $\begin{array}{c}\text { Green tea } \\
\text { extract }\end{array}$ & $250 \mathrm{mg}$ & Starch capsule & 10 & 10 \\
\hline $\begin{array}{l}\text { Hadi et al. } \\
2016\end{array}$ & 2016 & Iran & $\mathrm{DB} / \mathrm{R} / \mathrm{PC}$ & Soccer players & M & 3 & & & 22.6 & 22.82 & $\begin{array}{c}\text { Green tea } \\
\text { extract }\end{array}$ & $450 \mathrm{mg}$ & $\begin{array}{c}450 \mathrm{mg} \text { of } \\
\text { maltodextrin }\end{array}$ & 18 & 18 \\
\hline $\begin{array}{l}\text { Soeizi et al. } \\
2017\end{array}$ & 2017 & Iran & $\mathrm{SB} / \mathrm{R} / \mathrm{PC}$ & $\begin{array}{c}\beta-\text { Thalassemia } \\
\text { Major }\end{array}$ & $\mathrm{F} / \mathrm{M}$ & 8 & 23.1 & 24.2 & 20.9 & 19.42 & Green tea & $2500 \mathrm{mg}$ & Warm water & 26 & 26 \\
\hline
\end{tabular}


Table 1. Cont

\begin{tabular}{|c|c|c|c|c|c|c|c|c|c|c|c|c|c|c|c|}
\hline \multirow[b]{2}{*}{ Author } & \multirow{2}{*}{$\begin{array}{l}\text { Publication } \\
\text { Year }\end{array}$} & \multirow[b]{2}{*}{ Country } & \multirow{2}{*}{$\begin{array}{l}\text { Study } \\
\text { Design }\end{array}$} & \multirow[b]{2}{*}{ Participant } & \multirow[b]{2}{*}{ Sex } & \multirow{2}{*}{$\begin{array}{c}\text { Trial } \\
\text { Duration } \\
\text { (Week) }\end{array}$} & \multicolumn{2}{|c|}{ Means Age } & \multicolumn{2}{|c|}{ Means BMI } & \multicolumn{3}{|c|}{ Intervention } & \multicolumn{2}{|c|}{ Sample Size } \\
\hline & & & & & & & IG & CG & IG & CG & $\begin{array}{l}\text { Treatment } \\
\text { Group }\end{array}$ & $\begin{array}{c}\text { Green Tea Dose } \\
\text { (mg) }\end{array}$ & $\begin{array}{l}\text { Control } \\
\text { Group }\end{array}$ & IG & CG \\
\hline $\begin{array}{l}\text { Ribeiro Vaz } \\
\text { et al. } 2018\end{array}$ & 2018 & Brazil & $\mathrm{DB} / \mathrm{R} / \mathrm{PC}$ & $\begin{array}{c}\text { type } 1 \mathrm{DM} \text { or type } \\
2 \mathrm{DM}\end{array}$ & $\mathrm{F} / \mathrm{M}$ & 20 & 46.48 & 52.29 & 27.58 & 25.6 & $\begin{array}{c}\text { Green tea } \\
\text { extract }\end{array}$ & $1120 \mathrm{mg}$ & $\begin{array}{l}\text { cellulose } \\
\text { capsules }\end{array}$ & 27 & 28 \\
\hline $\begin{array}{l}\text { Venkatakrishnan } \\
\text { et al. } 2018\end{array}$ & 2018 & Taiwan & $\mathrm{DB} / \mathrm{R} / \mathrm{PC}$ & $\begin{array}{l}\text { Hypercholesterolemic } \\
\text { subjects }\end{array}$ & $\mathrm{F}$ & 12 & 45 & 45 & 31.39 & 28.81 & Green tea & $\begin{array}{c}600 \mathrm{~mL} \text { (780.6 mg } \\
\text { of catechin) }\end{array}$ & $\begin{array}{c}\text { Tea flavor } \\
\text { with very less } \\
\text { concentration } \\
\text { of catechin } \\
\text { and caffeine }\end{array}$ & 20 & 20 \\
\hline $\begin{array}{l}\text { Sadowska- } \\
\text { Krepa et al. } \\
2019\end{array}$ & 2019 & Poland & $\mathrm{RCT}$ & $\begin{array}{l}\text { Male students of } \\
\text { the physical } \\
\text { education }\end{array}$ & $\mathrm{M}$ & 6 & 22 & 23.1 & 23.73 & 24.72 & $\begin{array}{l}\text { Green tea } \\
\text { extract }\end{array}$ & $250 \mathrm{mg}$ & $\begin{array}{l}\text { Microcrystalline } \\
\text { cellulose, } \\
\text { magnesium } \\
\text { stearate and } \\
\text { maltodextrin }\end{array}$ & 8 & 8 \\
\hline $\begin{array}{c}\text { Sobhani et al. } \\
2020\end{array}$ & 2020 & Iran & $\mathrm{RCT}$ & $\begin{array}{c}\text { Healthy } \\
\text { individuals }\end{array}$ & $\mathrm{M}$ & 2 & 26.12 & 24 & 23.85 & 24.62 & $\begin{array}{c}\text { Green tea } \\
\text { extract }\end{array}$ & $\begin{array}{l}640 \mathrm{mg} \text { of } \\
\text { polyphenols }\end{array}$ & Maltodextrin & 8 & 7 \\
\hline $\begin{array}{l}\text { Bazyar et al. } \\
2020\end{array}$ & 2020 & Iran & $\mathrm{RCT}$ & T2DM patients & $\mathrm{F} / \mathrm{M}$ & 8 & 51.75 & 52.61 & 29.46 & 29.28 & $\begin{array}{c}\text { Green tea } \\
\text { extract }\end{array}$ & $600 \mathrm{mg}$ of EGCG & $\begin{array}{c}600 \mathrm{mg} \text { of } \\
\text { wheat flour }\end{array}$ & 22 & 22 \\
\hline
\end{tabular}

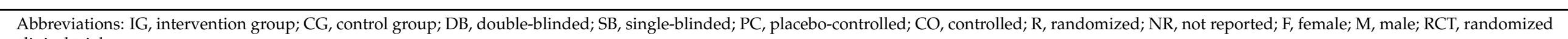
Abbreviations:
clinical trial.

Table 2. Quality assessment of the studies.

\begin{tabular}{|c|c|c|c|c|c|c|c|}
\hline Study & $\begin{array}{c}\text { Random Sequence } \\
\text { Generation }\end{array}$ & $\begin{array}{c}\text { Allocation } \\
\text { Concealment }\end{array}$ & $\begin{array}{c}\text { Blinding of } \\
\text { Participants and } \\
\text { Personnel }\end{array}$ & $\begin{array}{l}\text { Blinding of Outcome } \\
\text { Assessment }\end{array}$ & $\begin{array}{c}\text { Incomplete Outcome } \\
\text { Data }\end{array}$ & $\begin{array}{l}\text { Selective Outcome } \\
\text { Reporting }\end{array}$ & Other Sources of Bias \\
\hline Mousavi et al. (2013) & $\mathrm{U}$ & $\mathrm{H}$ & $\mathrm{H}$ & $\mathrm{U}$ & $\mathrm{L}$ & $\mathrm{L}$ & $\mathrm{U}$ \\
\hline Lasaite et al. (2014) & $\mathrm{U}$ & $\mathrm{L}$ & $\mathrm{L}$ & $\mathrm{U}$ & $\mathrm{L}$ & $\mathrm{L}$ & $\mathrm{L}$ \\
\hline Spadiene et al. (2014) & $\mathrm{U}$ & $\mathrm{L}$ & $\mathrm{L}$ & $\mathrm{U}$ & $\mathrm{L}$ & $\mathrm{L}$ & $\mathrm{L}$ \\
\hline Jówkoa et al. (2011) & $\mathrm{U}$ & $\mathrm{L}$ & $\mathrm{L}$ & $\mathrm{U}$ & $\mathrm{U}$ & $\mathrm{L}$ & $\mathrm{U}$ \\
\hline Sadowska-Krępa et al. (2019) & $\mathrm{U}$ & $\mathrm{L}$ & $\mathrm{H}$ & $\mathrm{U}$ & $\mathrm{L}$ & $\mathrm{L}$ & $\mathrm{U}$ \\
\hline Hadi et al. (2016) & $\mathrm{U}$ & $\mathrm{L}$ & $\mathrm{L}$ & $\mathrm{U}$ & $\mathrm{L}$ & $\mathrm{L}$ & $\mathrm{L}$ \\
\hline Mozaffari-Khosravi et al. (2014) & $\mathrm{U}$ & $\mathrm{H}$ & $\mathrm{U}$ & $\mathrm{U}$ & $\mathrm{U}$ & $\mathrm{L}$ & $\mathrm{U}$ \\
\hline
\end{tabular}


Table 2. Cont.

\begin{tabular}{|c|c|c|c|c|c|c|c|}
\hline Study & $\begin{array}{c}\text { Random Sequence } \\
\text { Generation }\end{array}$ & $\begin{array}{c}\text { Allocation } \\
\text { Concealment }\end{array}$ & $\begin{array}{c}\text { Blinding of } \\
\text { Participants and } \\
\text { Personnel }\end{array}$ & $\begin{array}{l}\text { Blinding of Outcome } \\
\text { Assessment }\end{array}$ & $\begin{array}{c}\text { Incomplete Outcome } \\
\text { Data }\end{array}$ & $\begin{array}{l}\text { Selective Outcome } \\
\text { Reporting }\end{array}$ & Other Sources of Bias \\
\hline Azizbeigi et al. (2019) & $\mathrm{U}$ & $\mathrm{U}$ & $\mathrm{H}$ & $\mathrm{U}$ & $\mathrm{U}$ & $\mathrm{L}$ & $\mathrm{U}$ \\
\hline Suliburska et al. (2012) & $\mathrm{L}$ & $\mathrm{L}$ & $\mathrm{L}$ & $\mathrm{L}$ & $\mathrm{L}$ & $\mathrm{L}$ & $\mathrm{L}$ \\
\hline Kuo et al. (2014) & $\mathrm{U}$ & $\mathrm{L}$ & $\mathrm{L}$ & $\mathrm{L}$ & $\mathrm{L}$ & $\mathrm{L}$ & $\mathrm{L}$ \\
\hline Soeizi et al. (2017) & $\mathrm{L}$ & $\mathrm{L}$ & $\mathrm{L}$ & $\mathrm{L}$ & $\mathrm{L}$ & $\mathrm{L}$ & $\mathrm{L}$ \\
\hline Sobhani et al. (2020) & $\mathrm{U}$ & $\mathrm{L}$ & $\mathrm{L}$ & $\mathrm{U}$ & $\mathrm{L}$ & $\mathrm{L}$ & $\mathrm{U}$ \\
\hline Bazyar et al. (2020) & $\mathrm{U}$ & $\mathrm{L}$ & $\mathrm{L}$ & $\mathrm{U}$ & $\mathrm{L}$ & $\mathrm{L}$ & $\mathrm{L}$ \\
\hline
\end{tabular}

Table 3. Subgroup analyses of green tea supplementation on oxidative stress biomarkers.

\begin{tabular}{|c|c|c|c|c|c|}
\hline & NO & WMD $(95 \% \mathrm{CI})$ & $p$ & P Heterogeneity & $\mathrm{I}^{2}$ \\
\hline \multicolumn{6}{|c|}{ Subgroup analyses of green tea supplementation on TAC concentrations. } \\
\hline Overall effect & 16 & $0.20(0.09,0.30)$ & $<0.001$ & $<0.001$ & $98.6 \%$ \\
\hline \multicolumn{6}{|l|}{ Trial duration (week) } \\
\hline$<8$ & 5 & $0.43(0.10,0.75)$ & 0.010 & $<0.001$ & $99.6 \%$ \\
\hline$\geq 8$ & 11 & $0.08(0.02,0.13)$ & 0.004 & $<0.001$ & $80.1 \%$ \\
\hline \multicolumn{6}{|l|}{ Type of green tea } \\
\hline Green tea extract & 12 & $0.23(0.03,0.42)$ & 0.019 & $<0.001$ & $98.9 \%$ \\
\hline Brewed green tea & 4 & $0.07(0.03,0.10)_{-}$ & $<0.001$ & 0.169 & $40.5 \%$ \\
\hline \multicolumn{6}{|l|}{ Sex } \\
\hline Both & 8 & $0.12(0.08,0.17)$ & $<0.001$ & 0.173 & $32.0 \%$ \\
\hline Male & 6 & $0.38(0.07,0.69)$ & 0.003 & $<0.001$ & $99.5 \%$ \\
\hline Female & 1 & $0.05(0.04,0.05)$ & 0.015 & - & - \\
\hline \multicolumn{6}{|c|}{ Intervention dose $(\mathrm{mg} / \mathrm{d})$} \\
\hline$\leq 400$ & 6 & $0.06(-0.01,0.13)$ & 0.132 & $<0.001$ & $87.1 \%$ \\
\hline$>400$ & 10 & $0.29(0.09,0.49)$ & 0.004 & $<0.001$ & $99.1 \%$ \\
\hline
\end{tabular}


Table 3. Cont.

\begin{tabular}{|c|c|c|c|c|c|}
\hline & NO & WMD $(95 \% \mathrm{CI})$ & $p$ & P Heterogeneity & $\mathrm{I}^{2}$ \\
\hline \multicolumn{6}{|l|}{ Health status } \\
\hline Healthy & 11 & $0.08(0.02,0.13)$ & 0.004 & $<0.001$ & $80.1 \%$ \\
\hline Unhealthy & 5 & $0.43(0.10,0.75)$ & 0.010 & $<0.001$ & $99.6 \%$ \\
\hline \multicolumn{6}{|l|}{ Baseline BMI $\left(\mathrm{kg} / \mathrm{m}^{2}\right)$} \\
\hline $18.5-24.9$ & 6 & $0.38(0.08,0.68)$ & 0.011 & $<0.001$ & $99.5 \%$ \\
\hline 25-29.9 & 4 & $0.11(0.07,0.15)$ & $<0.001$ & 0.387 & $1.1 \%$ \\
\hline$\geq 30$ & 5 & $0.06(-0.03,0.16)$ & 0.176 & $<0.001$ & $88.2 \%$ \\
\hline \multicolumn{6}{|c|}{ Subgroup analyses of green tea supplementation on MDA concentrations } \\
\hline Overall effect & 8 & $-0.00(-0.00,0.00)$ & 0.636 & $<0.001$ & $84.1 \%$ \\
\hline \multicolumn{6}{|l|}{ Trial duration (week) } \\
\hline$<8$ & 3 & $-0.08(-0.21,0.04)$ & 0.185 & 0.002 & $84.2 \%$ \\
\hline$\geq 8$ & 5 & $-0.00(-0.00,0.00)$ & 0.860 & $<0.001$ & $86.3 \%$ \\
\hline \multicolumn{6}{|l|}{ Type of green tea } \\
\hline Green tea extract & 4 & $-0.17(-0.43,0.09)$ & 0.202 & 0.004 & $77.1 \%$ \\
\hline Brewed green tea & 4 & $-0.00(-0.00,0.00)$ & 0.860 & $<0.001$ & $89.5 \%$ \\
\hline \multicolumn{6}{|l|}{ Sex } \\
\hline \multicolumn{6}{|l|}{ Intervention dose } \\
\hline$\leq 400$ & 2 & $-0.01(-0.02,0.00)$ & 0.215 & 0.530 & $0.0 \%$ \\
\hline$>400$ & 6 & $-0.00(-0.00,0.00)$ & 0.821 & $<0.001$ & $88.1 \%$ \\
\hline \multicolumn{6}{|l|}{ Health status } \\
\hline Healthy & 6 & $-0.00(-0.00,0.00)$ & 0.853 & $<0.001$ & $82.9 \%$ \\
\hline Unhealthy & 2 & $-0.20(-0.61,0.20)$ & 0.331 & $<0.001$ & $92.1 \%$ \\
\hline \multicolumn{6}{|l|}{ BMI } \\
\hline $18.5-24.9$ & 3 & $-0.46(-0.98,0.05)$ & 0.081 & $<0.001$ & $95.0 \%$ \\
\hline $25-29.9$ & 3 & $-0.00(-0.00,0.00)$ & 0.493 & 0.609 & $0.0 \%$ \\
\hline$\geq 30$ & 2 & $-0.12(-0.45,0.20)$ & 0.451 & 0.910 & $0.0 \%$ \\
\hline
\end{tabular}

Abbreviations: CI, confidence interval; WMD, weighted mean differences. 
Study

ID

WMD (95\% CI) Weight

Azizbeigi et al. 2009

Jowko et al. 2011

Bogdanski et al. 2012

Suliburska et al. 2012

Mousavi et al. 2013 (A)

Mousavi et al. 2013 (B)

Lasaite et al. 2014 (A)

Lasaite et al. 2014 (B)

Spadiene et al. 2014 (A)

Spadiene et al. 2014 (B)

Kuo et al. 2015

Hadi et al. 2016

Soeizi et al. 2017

Ribeiro Vaz et al. 2018

Venkatakrishnan et al. 2018

Sadowska-Krepa et al. 2019

Overall (I-squared $=98.6 \%, \mathrm{p}=0.000)$

NOTE: Weights are from random effects analysis

$$
-1.74
$$

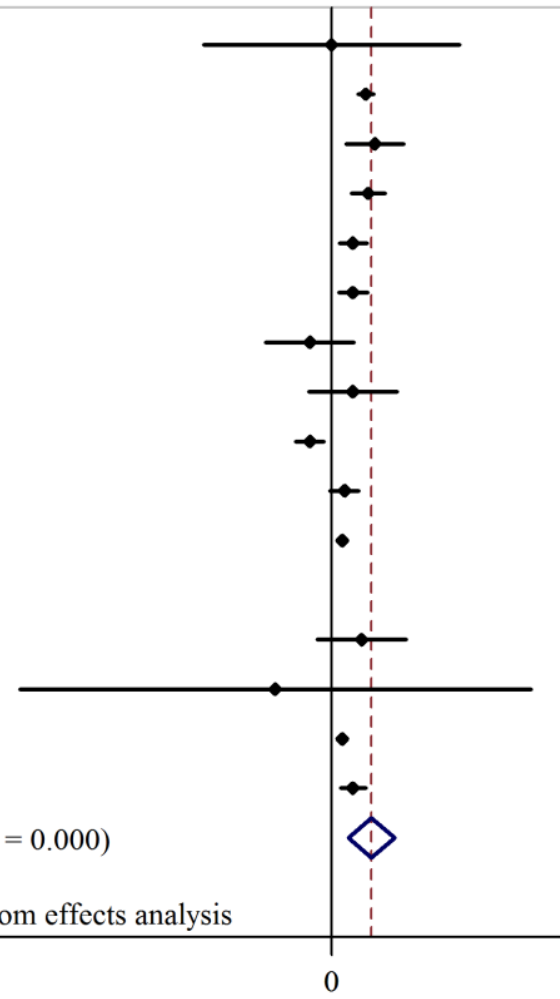

(A) TAC

Study

ID

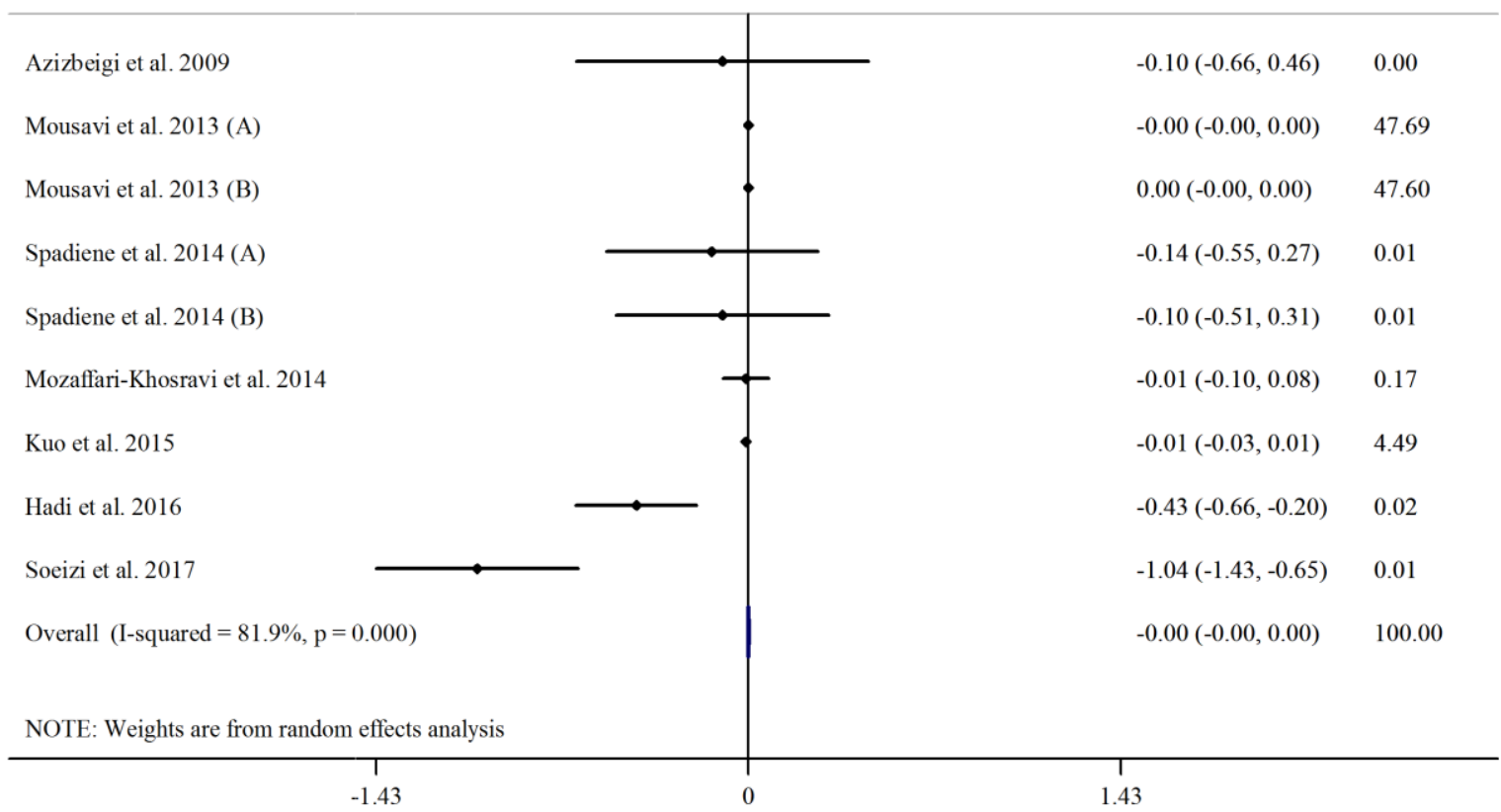

(B) MDA

Figure 2. Forest plot detailing weighted mean difference and $95 \%$ confidence intervals (CIs) for the effect of green tea consumption on; (A) TAC; (B) MDA.

\begin{tabular}{ll}
$0.00(-0.59,0.59)$ & 2.25 \\
$0.16(0.12,0.20)$ & 7.40 \\
$0.20(0.07,0.33)$ & 6.68 \\
$0.17(0.09,0.25)$ & 7.17 \\
$0.10(0.04,0.16)$ & 7.26 \\
$0.10(0.03,0.17)$ & 7.25 \\
$-0.10(-0.30,0.10)$ & 5.85 \\
$0.10(-0.10,0.30)$ & 5.85 \\
$-0.10(-0.17,-0.03)$ & 7.25 \\
$0.06(-0.01,0.13)$ & 7.25 \\
$0.05(0.03,0.07)$ & 7.43 \\
$1.64(1.54,1.74)$ & 7.02 \\
$0.14(-0.07,0.35)$ & 5.83 \\
$-0.26(-1.44,0.92)$ & 0.73 \\
$0.05(0.04,0.06)$ & 7.46 \\
$0.10(0.04,0.16)$ & 7.30 \\
$0.19(0.08,0.29)$ & 100.00 \\
\hline & \\
\hline
\end{tabular}

$\%$
WMD $(95 \% \mathrm{CI}) \quad$ Weight 


\subsubsection{Effect of Green Tea on MDA}

Eight clinical trials evaluated the effect of green tea on MDA. Pooled effect size from random effect model showed a nonsignificant decreasing effect of green tea supplementation on MDA (WMD: $-0.00 \mu \mathrm{mol} / \mathrm{L} ; 95 \% \mathrm{CI},-0.00,0.00, p=0.636$ ). There was significant heterogeneity between studies $\left(\mathrm{I}^{2}=84.1 \%, p<0.001\right)$ (Figure $\left.2 \mathrm{~B}\right)$. In addition, we performed subgroup analysis which did not indicate any changes in MDA following green tea supplementation; however, heterogeneity was reduced in interventions with less than $400 \mathrm{mg} /$ day $\left(\mathrm{I}^{2}=0.0 \%, p=0.530\right), \mathrm{BMI}=25-29.9\left(\mathrm{I}^{2}=0.0 \%, p=0.609\right)$ and $\mathrm{BMI} \geq 30$ $\left(\mathrm{I}^{2}=0.0 \%, p=0.910\right)($ Table 3$)$.

\subsubsection{Publication Bias and Sensitivity Analysis}

The sensitivity analysis indicated that removing any of the studies would not substantially change the effect of green tea supplementation on oxidative stress (Figure 2). We used Egger's weighted regression tests to explore the publication bias. There was no evidence of publication bias for studies examining the effect of green tea supplementation on MDA $(p=0.335$, Egger's test $)(p=0.711$ Begg test $)$, and TAC $(p=0.093$, Egger's test $)(p=0.893$ Begg test) (Figure 3A,B).

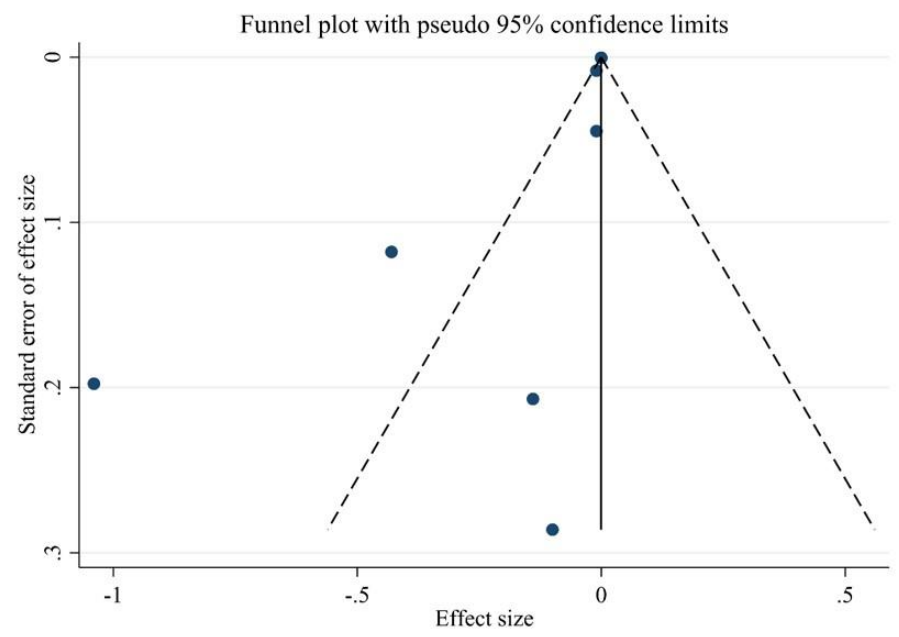

(A) TAC

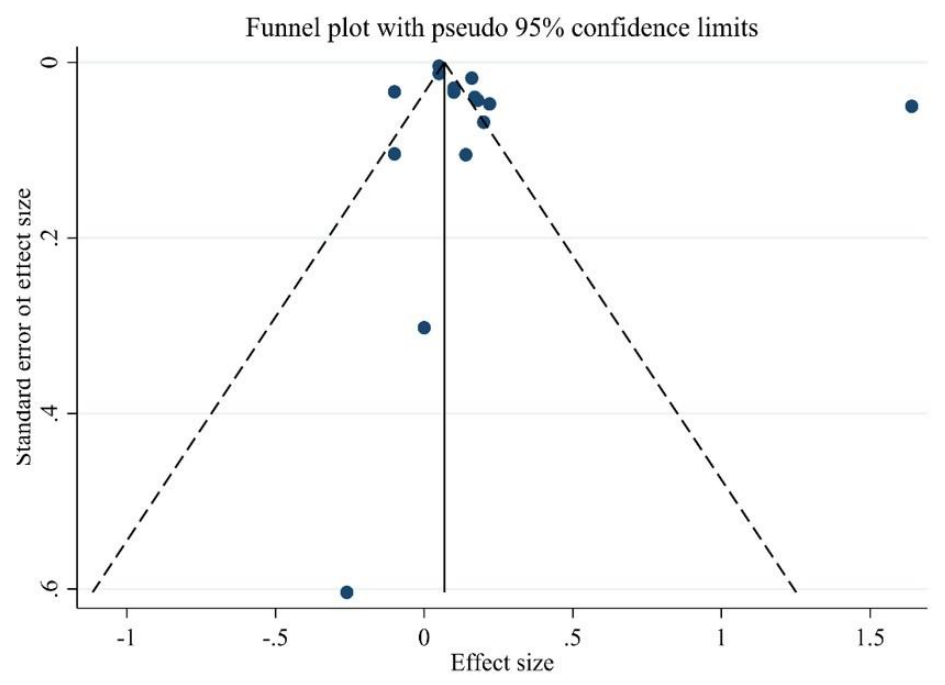

(B) MDA

Figure 3. Funnel plot for the effect of green tea consumption on; (A) TAC; (B) MDA. 
3.3.4. Non-Linear Dose-Response between Dose and Duration of Green Tea Supplementation and Antioxidant Status

Non-linear dose-response analysis was conducted between the dose and duration of green tea supplementation for TAC and MDA. Dose-response analysis indicated that green tea supplementation did not affect TAC and MDA concentrations (Figures 4A,B and 5A,B).

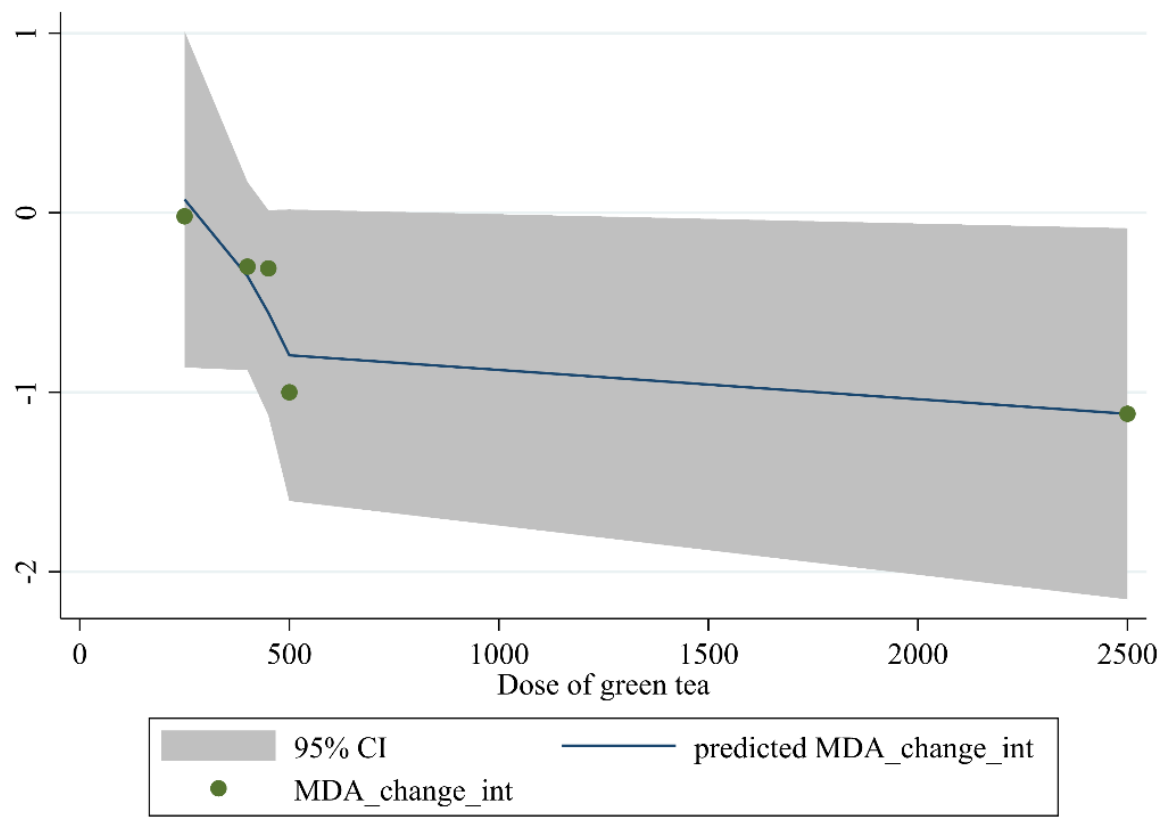

(A) TAC

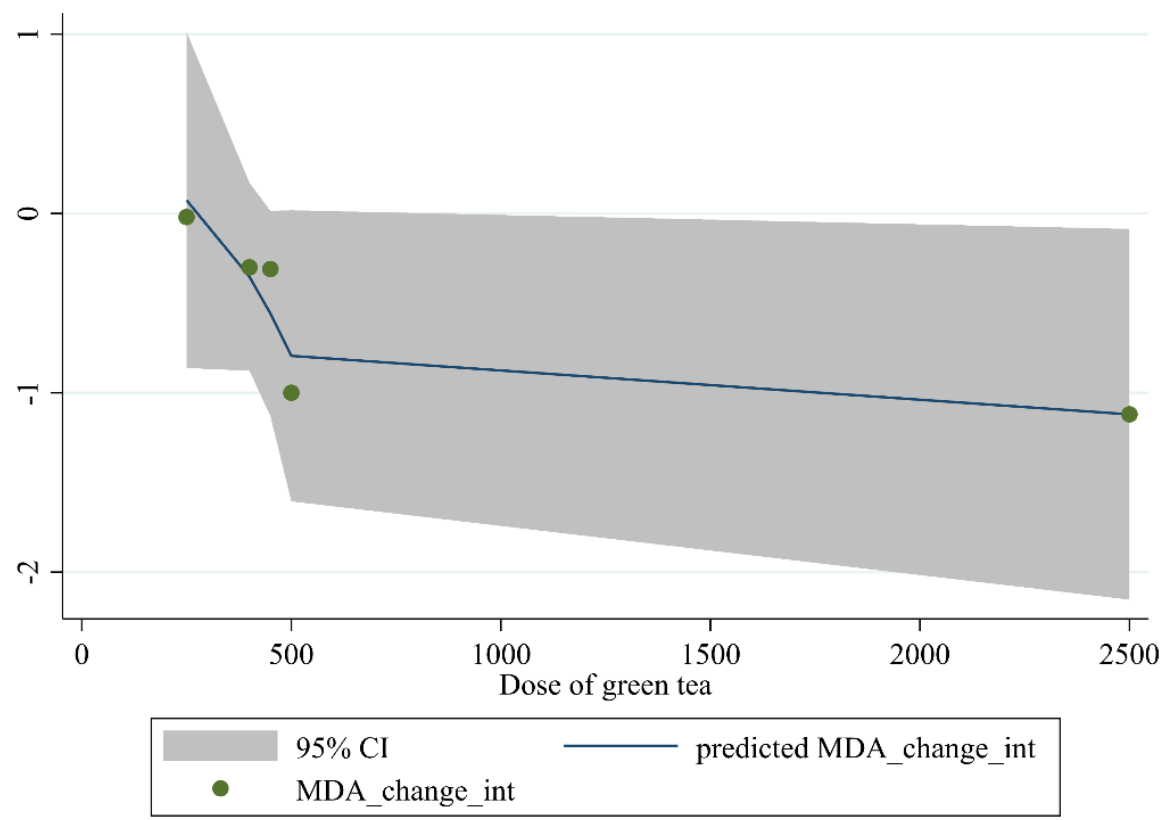

(B) MDA

Figure 4. Non-linear dose-response relations between dose of green tea (mg/day) and predicted changes in (A) TAC and (B) MDA. 


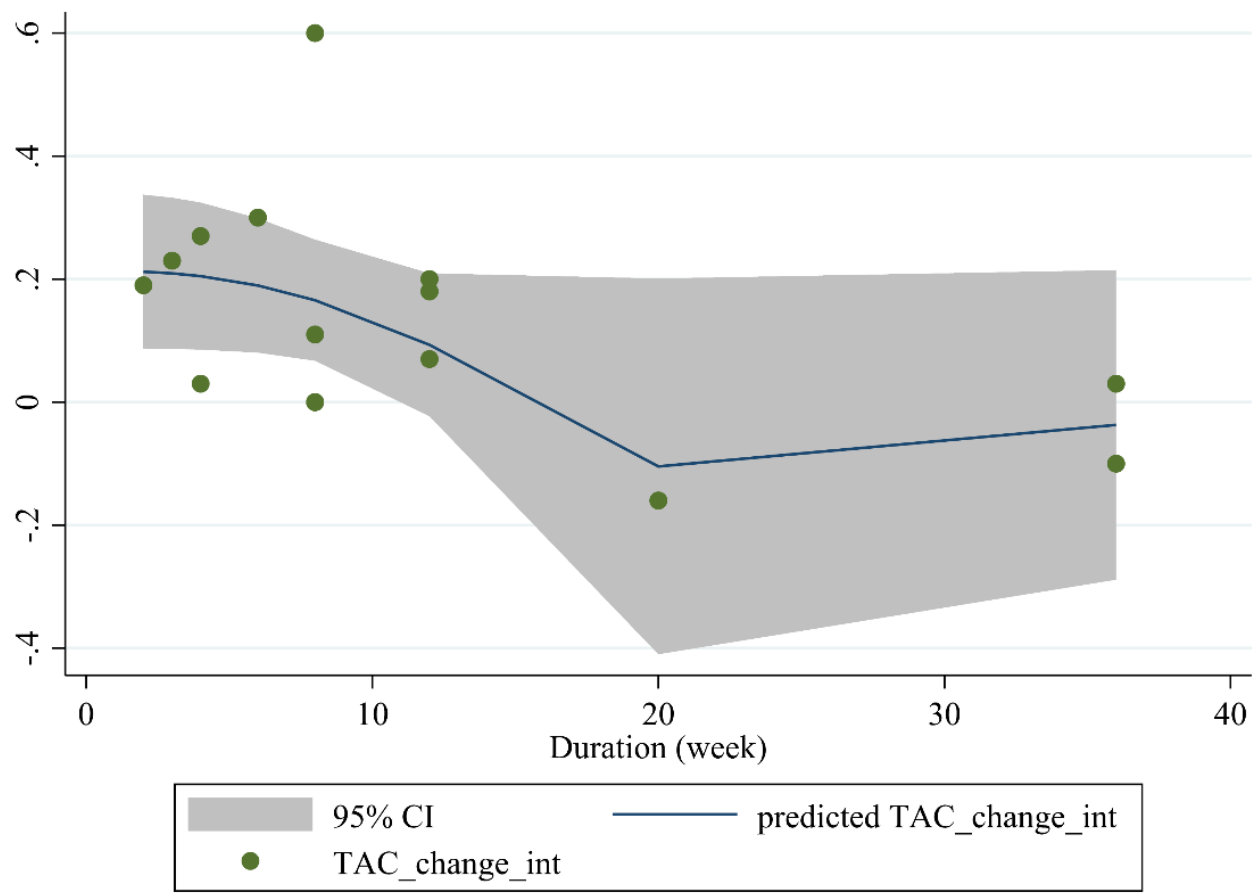

(A) TAC

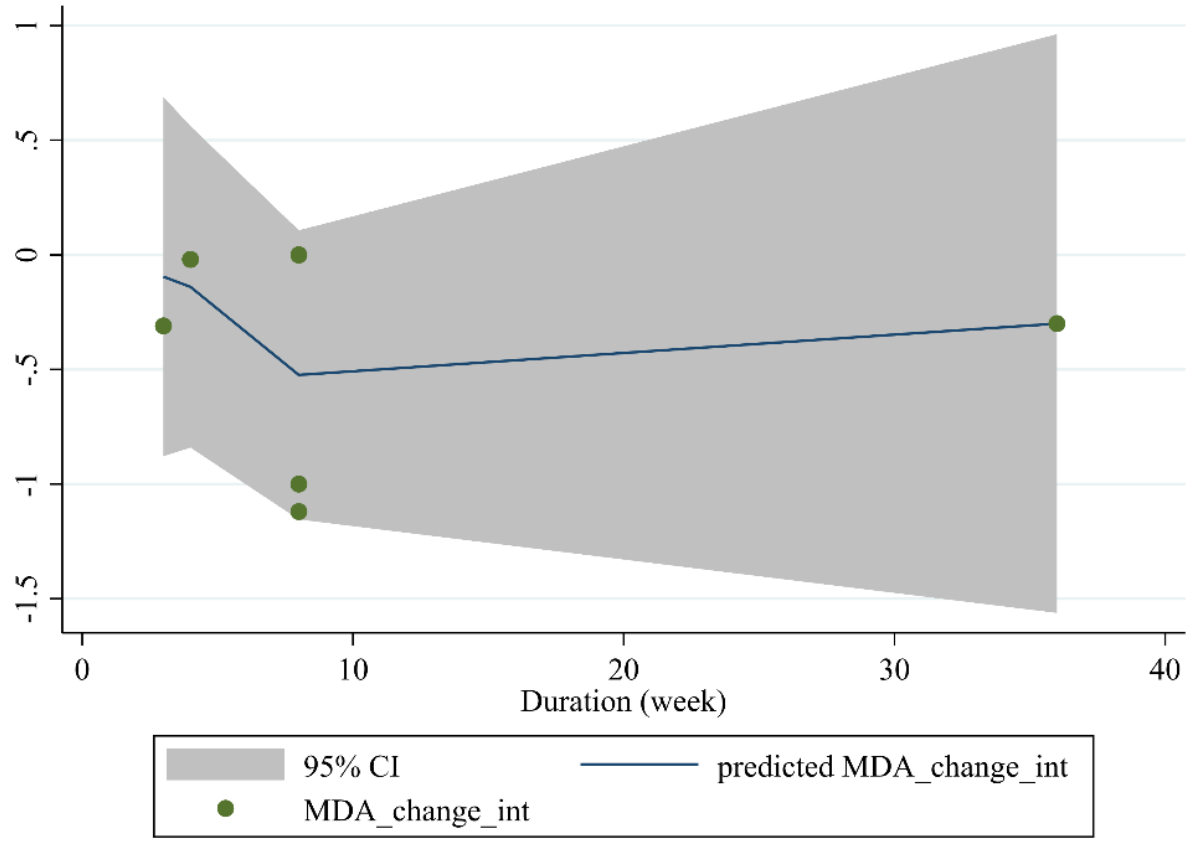

(B) MDA

Figure 5. Non-linear dose-response relations between duration of intervention (weeks) and predicted changes in (A) TAC and (B) MDA.

\subsubsection{Meta-Regression Analysis}

We performed meta-regression analysis to evaluate the association between the dose of green tea (mg/day) and the duration of the intervention (weeks) with antioxidant status. The results of the analysis revealed that green tea supplementation significantly changed MDA ( $r=-2117.18, p=0.017$ ) based on the dose of the intervention (Figure 6) and did not show a linear relationship between dose and duration with significant changes in TAC (Figures 7 and 8A,B). 


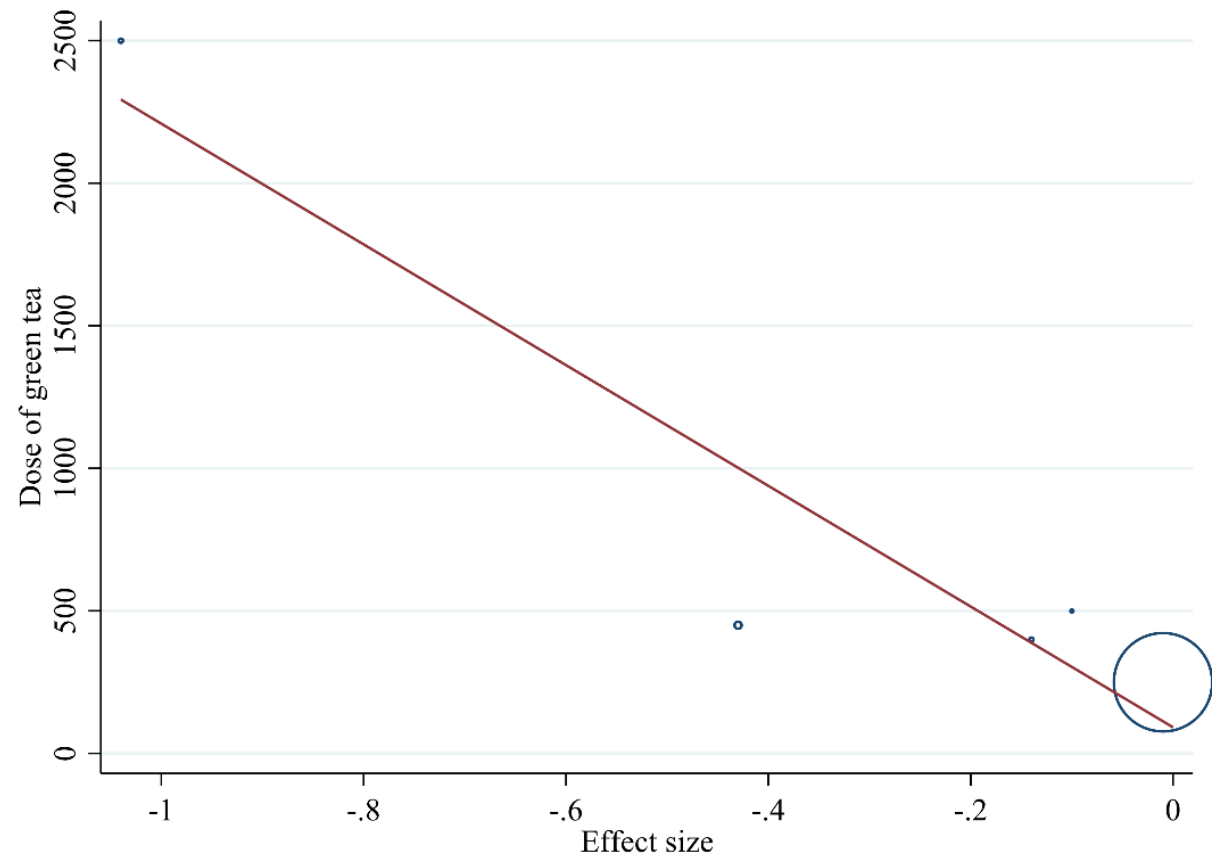

Figure 6. Random-effects meta-regression plot of the association between dose of green tea (mg/day) and MDA effect size.

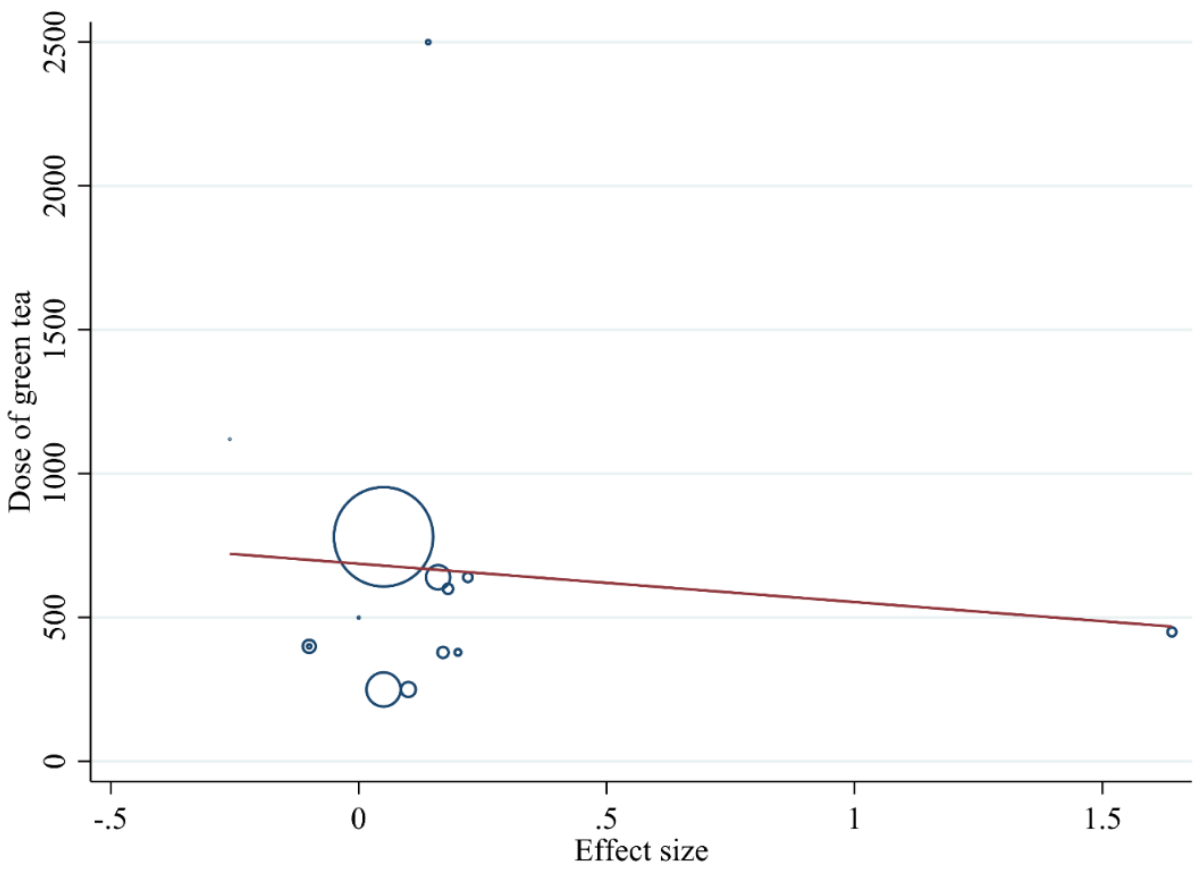

Figure 7. Random-effects meta-regression plot of the association between dose of green tea (mg/day) and TAC effect size. 


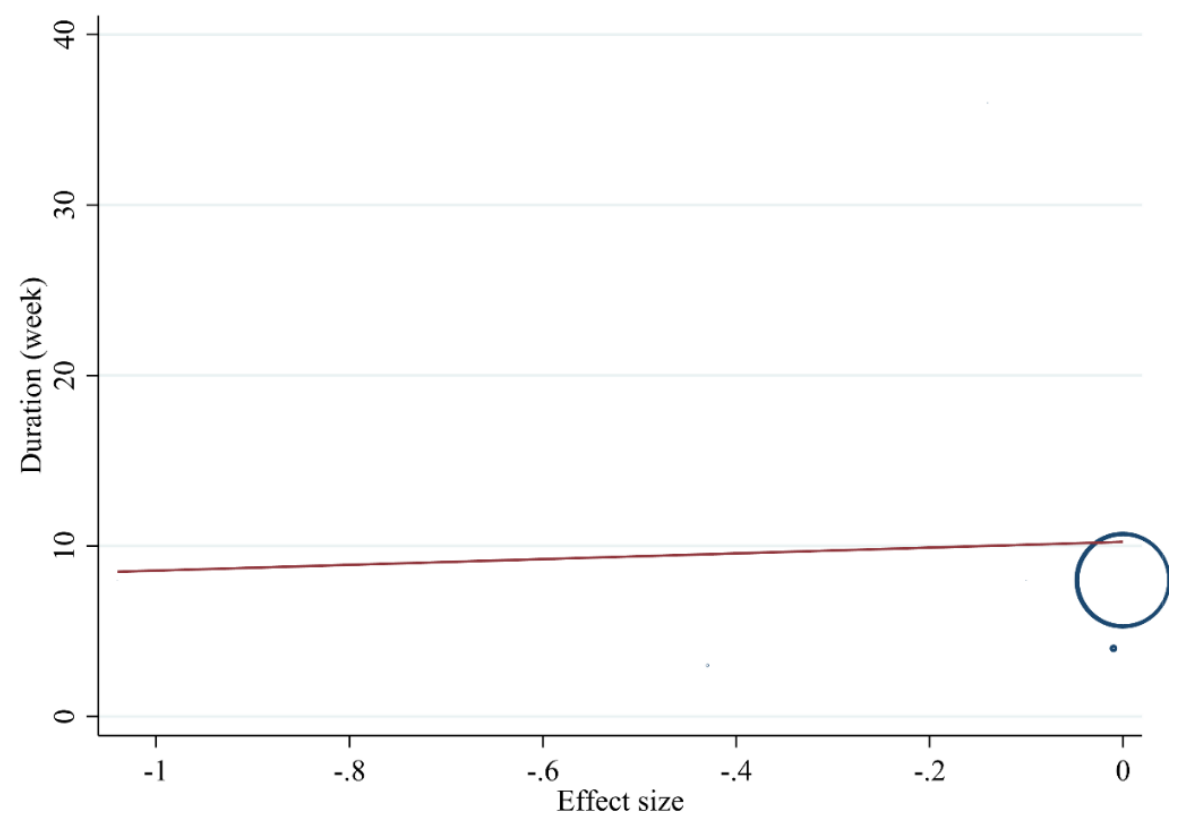

(A) MDA

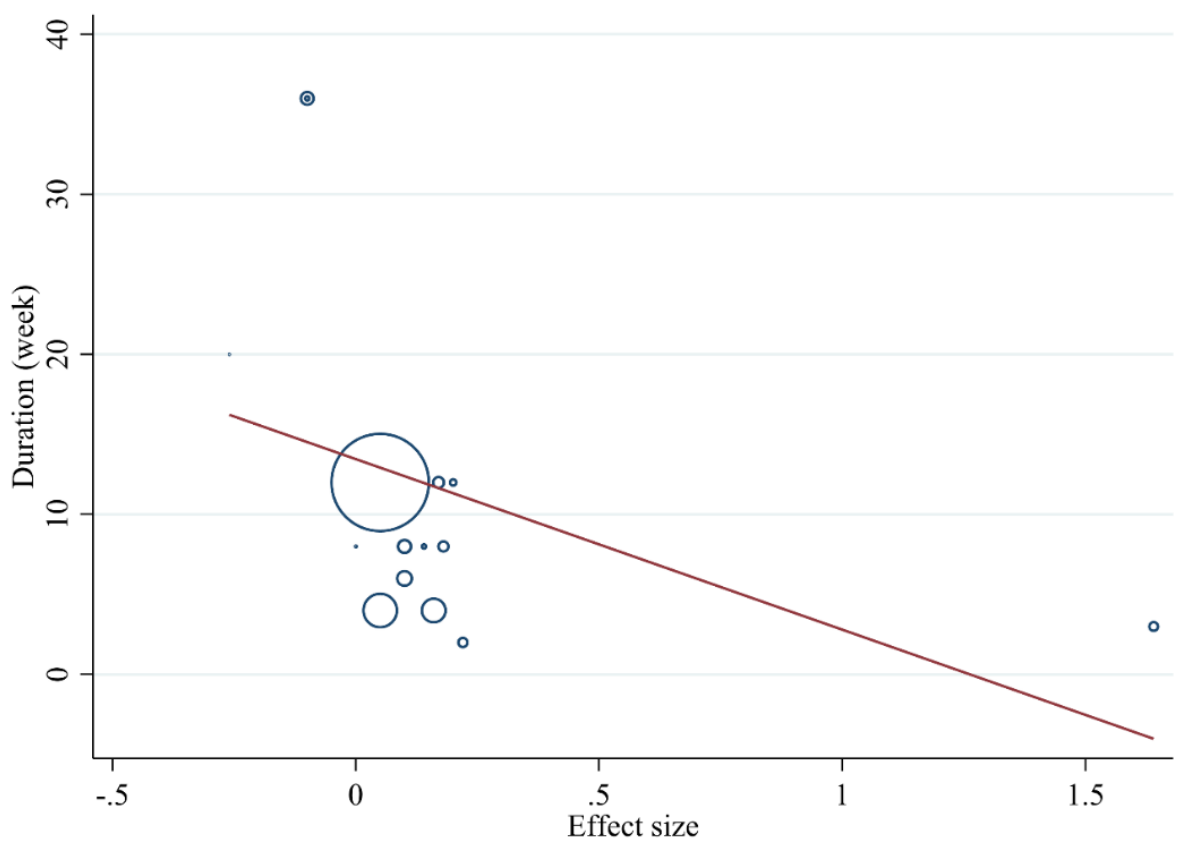

(B) TAC

Figure 8. Random-effects meta-regression plots of the association between duration of intervention (weeks) and effect size of (A) MDA and (B) TAC.

\section{Discussion}

To our knowledge, this is the first meta-analysis to evaluate the effects of green tea or green tea extract supplementation on oxidative stress (assessed TAC and circulating MDA concentrations). We identified 16 RCTs and 17 arms with 760 participants. These studies suggest that green tea and green tea extract supplementation were positively associated with TAC, but no association with MDA was observed in all subgroups. The association was independent of sex, BMI, duration, and green tea dosage.

Increased oxidative stress elevates the risk of chronic diseases, such as cardiovascular and end-stage renal disease, as it is related to the onset and progression of these adverse 
conditions $[11,12,62,63]$. A greater antioxidant intake has been previously related to a lower risk of disease mortality [62]. In general, Camellia sinensis and its tea are known as one of the significant sources of antioxidants such as flavonoids. It has also been shown that green tea is especially rich in a very strong antioxidant called epigallocatechin gallate $[63,64]$. For the first time, our research demonstrated a significant beneficial effect of green tea supplementation on TAC via a systematic review and meta-analysis, indicating the decreasing impact of this compound on oxidative stress development. The existence of catechins such as EGCG and ECG in green tea, which have numerous free hydroxyl groups, have significant beneficial effects on free radical scavenging activity, and therefore, can lessen the product of lipid peroxidation [65]. A growing body of evidence has suggested the possible role of green tea supplementation in enhancing the antioxidant capacity [30,66-69]. Additionally, catechin improves antioxidant capacity and may result in a significant decrease in lipid peroxidation production, which has been supported by the study of Lin et al. [66].

Several possible mechanisms are responsible for green tea polyphenols on increased plasma TAC. The vigorous antioxidative function of catechins is related to the dihydroxyl or trihydroxyl groups on the B-ring and the meta-5,7-dihydroxyl groups on the A ring. This function can also be heightened by the trihydroxyl structure in the D-ring (gallate) in EGCG and epicatechin gallate. Based on the polyphenolic structure, free radicals can be neutralized by Electron delocalization [67]. Moreover, reactive oxygen and nitric radicals can be excluded by tea catechins $[68,69]$.

The concentration of lipid peroxidation products representing oxidative stress across health and disease has not been adequately referenced [17-20]. In this present research, the MDA was proposed as an indicator of lipid peroxidation productions [70]. Regardless of the antioxidant activity of catechin, there are no consistent results on its effects on MDA. For instance, Hadi et al. in 2017 suggested that MDA concentrations were substantially subsided in athletes who consumed green tea extract [5]. This lowering effect of green tea on MDA was also confirmed by Noronha et al. 2019, which explains our findings [25]. Although we found that catechin has no significant effect on MDA concentrations in all subgroups, our meta-regression analysis demonstrated that green tea supplementation reduced MDA concentrations based on the dose of the intervention (mg/day).

On the contrary, several studies have shown no significant association between catechin and the value of MDA. The beneficial effects of green tea catechins in the elimination of ROS have been shown in vitro, although Heijnen et al. supported the idea that it may not be effective in vivo [71]. It has been observed that even with the highest dosage, the number of flavonoids and polyphenol in serum and intracellular are remarkably less than other types of antioxidant concentrations. It is also worth mentioning that some polyphenols, such as catechins, are the result of primitive polyphenols, and thus have poorer antioxidants levels than the parental polyphenols. It seems likely that dietary flavonoid actions as an antioxidant in vivo are not noticeable [72].

The present systematic review and meta-analysis is not without limitations. Most analyses had high levels of heterogeneity, but this was expected because included studies had different types of participants, doses, and intervention durations. In addition, there were not enough studies to evaluate all components of oxidative stress (MDA and TAC) in certain diseases. Moreover, our search was limited to RTCs published in English. Our meta-analytic work has several strengths, including RCTs and diverse participants (both healthy and diseased) from three different continents. Further well-designed RCTs are warranted to corroborate and expand on our outcomes, particularly in regions where green tea supplementation is not popular and in subjects with different diseases that may benefit from a decline in oxidative stress, such as those with cancer.

\section{Conclusions}

In conclusion, combined data from interventional studies showed green tea supplementation has a beneficial effect on TAC. Additionally, a linear inverse association between the dose of green tea supplementation and a significant change in MDA was reported. 
Green tea and green tea extract can be proposed as suitable drinks to increase antioxidant status in adults, which may decrease the risk and progression of chronic diseases related to higher oxidative stress levels.

Author Contributions: Conceptualization, N.R. and O.A.; methodology, N.R., O.A., L.S., F.G.; software, O.A.; validation, R.B., L.S. and F.G.; formal analysis, O.A.; investigation, R.B.; resources, N.R.; data curation, L.S.; writing—original draft preparation, N.R., L.S., R.B.; writing-review and editing, A.W., R.B., K.C., M.S., N.S. and K.S.; visualization, O.A.; supervision, R.B., K.M.; project administration, K.M. All authors have read and agreed to the published version of the manuscript.

Funding: This research received no external funding.

Conflicts of Interest: The authors declare no conflict of interest.

\section{References}

1. Aiyegoro, O.A.; Okoh, A.I. Preliminary phytochemical screening and In vitro antioxidant activities of the aqueous extract of Helichrysum longifolium DC. BMC Complement. Altern. Med. 2010, 10, 21. [CrossRef]

2. Jówko, E.; Sacharuk, J.; Bałasinska, B.; Wilczak, J.; Charmas, M.; Ostaszewski, P.; Charmas, R. Effect of a Single Dose of Green Tea Polyphenols on the Blood Markers of Exercise-Induced Oxidative Stress in Soccer Players. Int. J. Sport Nutr. Exerc. Metab. 2012, 22, 486-496. [CrossRef]

3. Henriksen, E.J.; Diamond-Stanic, M.K.; Marchionne, E.M. Oxidative stress and the etiology of insulin resistance and type 2 diabetes. Free Radic. Biol. Med. 2011, 51, 993-999. [CrossRef]

4. Jówko, E.; Sacharuk, J.; Bałasinska, B.; Ostaszewski, P.; Charmas, M.; Charmas, R. Green tea extract supplementation gives protection against exercise-induced oxidative damage in healthy men. Nutr. Res. 2011, 31, 813-821. [CrossRef]

5. Hadi, A.; Pourmasoumi, M.; Kafeshani, M.; Karimian, J.; Maracy, M.R.; Entezari, M. The Effect of Green Tea and Sour Tea (Hibiscus sabdariffa L.) Supplementation on Oxidative Stress and Muscle Damage in Athletes. J. Diet. Suppl. 2017, 14, 346-357. [CrossRef]

6. Wright, E., Jr.; Scism-Bacon, J.; Glass, L. Oxidative stress in type 2 diabetes: The role of fasting and postprandial glycaemia. Int. J. Clin. Pract. 2006, 60, 308-314. [CrossRef]

7. Atabek, M.E.; Vatansev, H.; Erkul, I. Oxidative stress in childhood obesity. J. Pediatr. Endocrinol. Metab. 2004, 17, 1063-1068. [CrossRef]

8. Kris-Etherton, P.M.; Hecker, K.D.; Bonanome, A.; Coval, S.M.; Binkoski, A.E.; Hilpert, K.F.; Griel, A.E.; Etherton, T.D. Bioactive compounds in foods: Their role in the prevention of cardiovascular disease and cancer. Am. J. Med. 2002, 113, 71-88. [CrossRef]

9. Azizbeigi, K.; Stannard, S.R.; Atashak, S. Green Tea Supplementation During Resistance Training Minimally Affects Systemic Inflammation and Oxidative Stress Indices in Obese Men. Jundishapur J. Nat. Pharm. Prod. in press. 2019. [CrossRef]

10. Vaz, S.R.; de Amorim, L.M.N.; de Nascimento, P.V.F.; Veloso, V.S.P.; Nogueira, M.S.; Castro, I.A.; Mota, J.F.; Botelho, P.B. Effects of green tea extract on oxidative stress and renal function in diabetic individuals: A randomized, double-blinded, controlled trial. $J$. Funct. Foods 2018, 46, 195-201. [CrossRef]

11. Nguyen, T.T.U.; Kim, H.W.; Kim, W. Effects of Probiotics, Prebiotics, and Synbiotics on Uremic Toxins, Inflammation, and Oxidative Stress in Hemodialysis Patients: A Systematic Review and Meta-Analysis of Randomized Controlled Trials. J. Clin. Med. 2021, 10, 4456. [CrossRef]

12. Liakopoulos, V.; Roumeliotis, S.; Gorny, X.; Dounousi, E.; Mertens, P.R. Oxidative Stress in Hemodialysis Patients: A Review of the Literature. Oxidative Med. Cell. Longev. 2017, 2017, 3081856. [CrossRef]

13. Lasaite, L.; Spadiene, A.; Savickiene, N.; Skesters, A.; Silova, A. The Effect of Ginkgo biloba and Camellia sinensis Extracts on Psychological State and Glycemic Control in Patients with Type 2 Diabetes Mellitus. Nat. Prod. Commun. 2014, 9, 1345-1350. [CrossRef]

14. Tresserra-Rimbau, A. Dietary Polyphenols and Human Health. Nutrition 2020, 12, 2893. [CrossRef]

15. Thielecke, F.; Boschmann, M. The potential role of green tea catechins in the prevention of the metabolic syndrome-A review. Phytochemistry 2009, 70, 11-24. [CrossRef] [PubMed]

16. Phung, O.J.; Baker, W.; Matthews, L.J.; Lanosa, M.; Thorne, A.; Coleman, C. Effect of green tea catechins with or without caffeine on anthropometric measures: A systematic review and meta-analysis. Am. J. Clin. Nutr. 2009, 91, 73-81. [CrossRef]

17. Vahabzadeh, Z.; Molodi, M.; Nikkho, B.; Saghebjoo, M.; Saedmocheshi, S.; Zamani, F.; Roshani, Y.; Babanzadeh, S. Aerobic training and hydroalcoholic extracts of green tea improve pro-oxidant-antioxidant balance and histopathological score in the N-methyl-N-nitrosourea-induced prostate cancer model of rat. EXCLI J. 2020, 19, 762-772.

18. Sirichaiwetchakoon, K.; Lowe, G.M.; Eumkeb, G. The Free Radical Scavenging and Anti-Isolated Human LDL Oxidation Activities of Pluchea indica (L.) Less. Tea Compared to Green Tea (Camellia sinensis). BioMed Res. Int. 2020, 2020, 4183643. [CrossRef]

19. Ye, Q.; Ye, L.; Xu, X.; Huang, B.; Zhang, X.; Zhu, Y.; Chen, X. Epigallocatechin-3-gallate suppresses 1-methyl-4-phenyl-pyridineinduced oxidative stress in PC12 cells via the SIRT1/PGC-1 $\alpha$ signaling pathway. BMC Complement. Altern. Med. $2012,12,82$. [CrossRef] 
20. Ayissi, V.B.O.; Ebrahimi, A.; Schluesenner, H. Epigenetic effects of natural polyphenols: A focus on SIRT1-mediated mechanisms. Mol. Nutr. Food Res. 2014, 58, 22-32. [CrossRef]

21. Panza, V.S.P.; Wazlawik, E.; Schütz, G.R.; Comin, L.; Hecht, K.C.; da Silva, E.L. Consumption of green tea favorably affects oxidative stress markers in weight-trained men. Nutrition 2008, 24, 433-442. [CrossRef] [PubMed]

22. Basu, A.; Betts, N.M.; Mulugeta, A.; Tong, C.; Newman, E.; Lyons, T.J. Green tea supplementation increases glutathione and plasma antioxidant capacity in adults with the metabolic syndrome. Nutr. Res. 2013, 33, 180-187. [CrossRef] [PubMed]

23. Mousavi, A.; Vafa, M.; Neyestani, T.R.; Khamseh, M.; Hoseini, F. The effects of green tea consumption on metabolic and anthropometric indices in patients with Type 2 diabetes. J. Res. Med. Sci. 2013, 18, 1080-1086.

24. Koutelidakis, A.E.; Rallidis, L.; Koniari, K.; Panagiotakos, D.; Komaitis, M.; Zampelas, A.; Anastasiou-Nana, M.; Kapsokefalou, M. Effect of green tea on postprandial antioxidant capacity, serum lipids, C-reactive protein and glucose levels in patients with coronary artery disease. Eur. J. Nutr. 2013, 53, 479-486. [CrossRef]

25. Noronha, N.Y.; Pinhel, M.A.; Nicoletti, C.F.; Quinhoneiro, D.C.G.; Pinhanelli, V.C.; de Oliveira, B.A.; Nonino, C.B. Green tea supplementation improves oxidative stress biomarkers and modulates IL-6 circulating levels in obese women. Nutr. Hosp. Organo Of. Soc. Española Nutr. Parenter. Enter. 2019, 36, 583-588.

26. Moher, D.; Liberati, A.; Tetzlaff, J.; Altman, D.G. Preferred Reporting Items for Systematic Reviews and Meta-Analyses: The PRISMA Statement. Ann. Intern. Med. 2009, 151, 264-269. [CrossRef]

27. Richardson, W.S.; Wilson, M.C.; Nishikawa, J.; Hayward, R.S. The well-built clinical question: A key to evidence-based decisions. ACP J. Club 1995, 123, A12-A13. [CrossRef]

28. Higgins, J.P.T.; Altman, D.G.; Gøtzsche, P.C.; Jüni, P.; Moher, D.; Oxman, A.D.; Savović, J.; Schulz, K.F.; Weeks, L.; Sterne, J.A.C.; et al. The Cochrane Collaboration's tool for assessing risk of bias in randomised trials. BMJ 2011, 343 , d5928. [CrossRef]

29. Soeizi, E.; Rafraf, M.; Asghari-Jafarabadi, M.; Ghaffari, A.; Rezamand, A.; Doostan, F. Effects of green tea on serum iron parameters and antioxidant status in patients with $\beta$-thalassemia major. Pharm. Sci. 2017, 23, 27-36. [CrossRef]

30. Suliburska, J.; Bogdanski, P.; Szulinska, M.; Stepien, M.; Pupek-Musialik, D.; Jabłecka, A. Effects of Green Tea Supplementation on Elements, Total Antioxidants, Lipids, and Glucose Values in the Serum of Obese Patients. Biol. Trace Element Res. 2012, 149, 315-322. [CrossRef]

31. Spadiene, A.; Savickiene, N.; Ivanauskas, L.; Jakštas, V.; Skesters, A.; Silova, A.; Rodovicius, H. Antioxidant effects of Camellia sinensis L. extract in patients with type 2 diabetes. J. Food Drug Anal. 2014, 22, 505-511. [CrossRef]

32. Sadowska-Krępa, E.; Domaszewski, P.; Pokora, I.; Żebrowska, A.; Gdańska, A.; Podgórski, T. Effects of medium-term green tea extract supplementation combined with CrossFit workout on blood antioxidant status and serum brain-derived neurotrophic factor in young men: A pilot study. J. Int. Soc. Sports Nutr. 2019, 16, 1-9. [CrossRef]

33. Mozaffari-Khosravi, H.; Ahadi, Z.; Tafti, M.F. The Effect of Green Tea versus Sour Tea on Insulin Resistance, Lipids Profiles and Oxidative Stress in Patients with Type 2 Diabetes Mellitus: A Randomized Clinical Trial. Iran. J. Med. Sci. $2014,39,424-432$.

34. Kuo, Y.-C.; Lin, J.-C.; Bernard, J.R.; Liao, Y.-H. Green tea extract supplementation does not hamper endurance-training adaptation but improves antioxidant capacity in sedentary men. Appl. Physiol. Nutr. Metab. 2015, 40, 990-996. [CrossRef]

35. Venkatakrishnan, K.; Chiu, H.-F.; Cheng, J.-C.; Chang, Y.-H.; Lu, Y.-Y.; Han, Y.-C.; Shen, Y.-C.; Tsai, K.-S.; Wang, C.-K. Comparative studies on the hypolipidemic, antioxidant and hepatoprotective activities of catechin-enriched green and oolong tea in a double-blind clinical trial. Food Funct. 2018, 9, 1205-1213. [CrossRef]

36. Sobhani, V.; Mehrtash, M.; Shirvani, H.; Fasihi-Ramandi, M. Effects of Short-Term Green Tea Extract Supplementation on VO 2 Max and Inflammatory and Antioxidant Responses of Healthy Young Men in a Hot Environment. Int. J. Prev. Med. 2020, 11, 170.

37. Bazyar, H.; Hosseini, S.A.; Saradar, S.; Mombaini, D.; Allivand, M.; Labibzadeh, M.; Alipour, M. Effects of epigallocatechin-3gallate of Camellia sinensis leaves on blood pressure, lipid profile, atherogenic index of plasma and some inflammatory and antioxidant markers in type 2 diabetes mellitus patients: A clinical trial. J. Complement. Integr. Med. 2021, 18, 405-411. [CrossRef]

38. Borenstein, M.; Hedges, L.; Higgins, J.; Rothstein, H.R. References; Wiley Online Library: Hoboken, NJ, USA, 2009.

39. Hozo, S.P.; Djulbegovic, B.; Hozo, I. Estimating the mean and variance from the median, range, and the size of a sample. BMC Med. Res. Methodol. 2005, 5, 13. [CrossRef]

40. Moura, B.M.; Panza, V.P.; Brunetta, H.S.; Tamborindeguy, A.C.; De Oliveira, M.V.; Sakugawa, R.L.; Nunes, E.A.; Da Silva, E.L.; Diefenthaeler, F. Effect of mate tea consumption on rapid force production after eccentric exercise: A randomized, controlled, crossover study. Sport Sci. Health 2020, 16, 571-581. [CrossRef]

41. Cazzola, R.; Rondanelli, M. N-Oleoyl-Phosphatidyl-Ethanolamine and Epigallo Catechin-3-Gallate Mitigate Oxidative Stress in Overweight and Class I Obese People on a Low-Calorie Diet. J. Med. Food 2020, 23, 319-325. [CrossRef] [PubMed]

42. Agarwal, A.; Prasad, R.; Jain, A. Effect of green tea extract (catechins) in reducing oxidative stress seen in patients of pulmonary tuberculosis on DOTS Cat I regimen. Phytomedicine 2010, 17, 23-27. [CrossRef] [PubMed]

43. Alexopoulos, N.; Vlachopoulos, C.; Aznaouridis, K.; Baou, K.; Vasiliadou, C.; Pietri, P.; Xaplanteris, P.; Stefanadi, E.; Stefanadis, C. The acute effect of green tea consumption on endothelial function in healthy individuals. Eur. J. Cardiovasc. Prev. Rehabil. 2008, 15, 300-305. [CrossRef]

44. Basu, A.; Sanchez, K.; Leyva, M.J.; Wu, M.; Betts, N.M.; Aston, C.E.; Lyons, T.J. Green Tea Supplementation Affects Body Weight, Lipids, and Lipid Peroxidation in Obese Subjects with Metabolic Syndrome. J. Am. Coll. Nutr. 2010, 29, 31-40. [CrossRef] [PubMed] 
45. Chiu, H.-F.; Lin, T.-Y.; Shen, Y.-C.; Venkatakrishnan, K.; Wang, C.-K. Improvement of green tea polyphenol with milk on skin with respect to antioxidation in healthy adults: A double-blind placebo-controlled randomized crossover clinical trial. Food Funct. 2016, 7, 893-901. [CrossRef]

46. Arab, H.; Mahjoub, S.; Hajian-Tilaki, K.; Moghadasi, M. The effect of green tea consumption on oxidative stress markers and cognitive function in patients with Alzheimer's disease: A prospective intervention study. Casp. J. Intern. Med. 2016, 7, 188-194.

47. Erba, D.; Riso, P.; Bordoni, A.; Foti, P.; Biagi, P.; Testolin, G. Effectiveness of moderate green tea consumption on antioxidative status and plasma lipid profile in humans. J. Nutr. Biochem. 2005, 16, 144-149. [CrossRef]

48. Freese, R.; Basu, S.; Hietanen, E.; Nair, J.; Nakachi, K.; Bartsch, H.; Mutanen, M. Green tea extract decreases plasma malondialdehyde concentration but does not affect other indicators of oxidative stress, nitric oxide production, or hemostatic factors during a high-linoleic acid diet in healthy females. Eur. J. Nutr. 1999, 38, 149-157. [CrossRef]

49. Hirano-Ohmori, R.; Takahashi, R.; Momiyama, Y.; Taniguchi, H.; Yonemura, A.; Tamai, S.; Umegaki, K.; Nakamura, H.; Kondo, K.; Ohsuzu, F. Green Tea Consumption and Serum Malondialdehyde-Modified LDL Concentrations in Healthy Subjects. J. Am. Coll. Nutr. 2005, 24, 342-346. [CrossRef]

50. Ide, K.; Yamada, H.; Takuma, N.; Kawasaki, Y.; Harada, S.; Nakase, J.; Ukawa, Y.; Sagesaka, Y.M. Effects of green tea consumption on cognitive dysfunction in an elderly population: A randomized placebo-controlled study. Nutr. J. 2015, 15, 1-9. [CrossRef]

51. Li, L.; Chen, C.-Y.O.; Aldini, G.; Johnson, E.J.; Rasmussen, H.; Yoshida, Y.; Niki, E.; Blumberg, J.B.; Russell, R.M.; Yeum, K.-J. Supplementation with lutein or lutein plus green tea extracts does not change oxidative stress in adequately nourished older adults. J. Nutr. Biochem. 2010, 21, 544-549. [CrossRef] [PubMed]

52. Liu, P.; Zhang, M.; Xie, X.; Jin, J.; Holman, C.D.J. Green tea consumption and glutathione S-transferases genetic polymorphisms on the risk of adult leukemia. Eur. J. Nutr. 2015, 56, 603-612. [CrossRef]

53. Young, J.F.; Dragstedt, L.O.; Haraldsdóttir, J.; Daneshvar, B.; Kall, M.A.; Loft, S.; Nilsson, L.; Nielsen, S.E.; Mayer, B.; Skibsted, L.H.; et al. Green tea extract only affects markers of oxidative status postprandially: Lasting antioxidant effect of flavonoid-free diet. Br. J. Nutr. 2002, 87, 343-355. [CrossRef]

54. Narotzki, B.; Reznick, A.Z.; Mitki, T.; Aizenbud, D.; Levy, Y. Green Tea Drinking Improves Erythrocytes and Saliva Oxidative Status in the Elderly. In Advances in Experimental Medicine and Biology; Springer: New York, NY, USA, 2014; Volume 832, pp. 25-33.

55. Nagao, T.; Komine, Y.; Soga, S.; Meguro, S.; Hase, T.; Tanaka, Y.; Tokimitsu, I. Ingestion of a tea rich in catechins leads to a reduction in body fat and malondialdehyde-modified LDL in men. Am. J. Clin. Nutr. 2005, 81, 122-129. [CrossRef]

56. Sami, G.; Khoshraftar, E.; Shayesteh, T.H.; Ranjbar, A. The effects of Chamomile tea on antioxidative biomarkers in operating room staff. J. HerbMed Pharmacol. 2015, 4, 98-101.

57. Sapper, T.N.; Mah, E.; Ahn-Jarvis, J.; McDonald, J.D.; Chitchumroonchokchai, C.; Reverri, E.J.; Vodovotz, Y.; Bruno, R.S. A green tea-containing starch confection increases plasma catechins without protecting against postprandial impairments in vascular function in normoglycemic adults. Food Funct. 2016, 7, 3843-3853. [CrossRef]

58. Lowe, G.M.; Gana, K.; Rahman, K. Dietary supplementation with green tea extract promotes enhanced human leukocyte activity. J. Complement. Integr. Med. 2015, 12, 277-282. [CrossRef]

59. Suraphad, P.; Suklaew, P.O.; Ngamukote, S.; Adisakwattana, S.; Mäkynen, K. The Effect of Isomaltulose Together with Green Tea on Glycemic Response and Antioxidant Capacity: A Single-Blind, Crossover Study in Healthy Subjects. Nutrition 2017, 9, 464. [CrossRef]

60. Hrishi, T.; Kundapur, P.; Naha, A.; Thomas, B.; Kamath, S.; Bhat, G. Effect of adjunctive use of green tea dentifrice in periodontitis patients-A Randomized Controlled Pilot Study. Int. J. Dent. Hyg. 2015, 14, 178-183. [CrossRef]

61. Da Silva, W.; Machado, Á.S.; Souza, M.A.; Mello-Carpes, P.B.; Carpes, F.P. Effect of green tea extract supplementation on exercise-induced delayed onset muscle soreness and muscular damage. Physiol. Behav. 2018, 194, 77-82. [CrossRef]

62. Parohan, M.; Anjom-Shoae, J.; Nasiri, M.; Khodadost, M.; Khatibi, S.R.; Sadeghi, O. Dietary total antioxidant capacity and mortality from all causes, cardiovascular disease and cancer: A systematic review and dose-response meta-analysis of prospective cohort studies. Eur. J. Nutr. 2019, 58, 2175-2189. [CrossRef]

63. Vinson, J.A.; Dabbagh, Y.A.; Serry, M.M.; Jang, J. Plant Flavonoids, Especially Tea Flavonols, Are Powerful Antioxidants Using an in Vitro Oxidation Model for Heart Disease. J. Agric. Food Chem. 1995, 43, 2800-2802. [CrossRef]

64. Prior, R.L.; Cao, G. Antioxidant Capacity and Polyphenols Components of Teas: Implications for Altering In Vivo Antioxidant Status. Exp. Biol. Med. 1999, 220, 255-261. [CrossRef]

65. Bogdanski, P.; Suliburska, J.; Szulinska, M.; Stepien, M.; Pupek-Musialik, D.; Jablecka, A. Green tea extract reduces blood pressure, inflammatory biomarkers, and oxidative stress and improves parameters associated with insulin resistance in obese, hypertensive patients. Nutr. Res. 2012, 32, 421-427. [CrossRef] [PubMed]

66. Lin, C.-C.; Li, C.-W.; Shih, Y.-T.; Chuang, L.-T. Antioxidant and Anti-Inflammatory Properties of Lower-Polymerized Polyphenols in Oolong Tea. Int. J. Food Prop. 2013, 17, 752-764. [CrossRef]

67. Yang, C.S.; Lambert, J.D.; Sang, S. Antioxidative and anti-carcinogenic activities of tea polyphenols. Arch. Toxicol. 2008, 83, 11-21. [CrossRef]

68. Ahn, H.Y.; Kim, C.H. Epigallocatechin-3-gallate Regulates Inducible Nitric Oxide Synthase Expression in Human Umbilical Vein Endothelial Cells. Lab. Anim. Res. 2011, 27, 85-90. [CrossRef]

69. Tayama, Y.; Sugihara, K.; Sanoh, S.; Miyake, K.; Morita, S.; Kitamura, S.; Ohta, S. Effect of Tea Beverages on Aldehyde Oxidase Activity. Drug Metab. Pharmacokinet. 2011, 26, 94-101. [CrossRef] 
70. Coimbra, S.; Castro, E.; Rocha-Pereira, P.; Rebelo, I.; Rocha, S.; Santos-Silva, A. The effect of green tea in oxidative stress. Clin. Nutr. 2006, 25, 790-796. [CrossRef]

71. Heijnen, C.; Haenen, G.; van Acker, F.; van der Vijgh, W.; Bast, A. Flavonoids as peroxynitrite scavengers: The role of the hydroxyl groups. Toxicol. Vitr. 2001, 15, 3-6. [CrossRef]

72. Kim, W.; Yang, H.J.; Youn, H.; Yun, Y.J.; Seong, K.M.; Youn, B. Myricetin inhibits Akt survival signaling and induces Bad-mediated apoptosis in a low dose ultraviolet (UV)-B-irradiated HaCaT human immortalized keratinocytes. J. Radiat. Res. 2010, 51, 285-296. [CrossRef] 